ESAIM: COCV 21 (2015) 757-788

DOI: $10.1051 / \mathrm{cocv} / 2014046$
ESAIM: Control, Optimisation and Calculus of Variations

www.esaim-cocv.org

\title{
ROUGH WALL EFFECT ON MICRO-SWIMMERS *
}

\author{
DAVID GÉRARD-VARET ${ }^{1}$ AND LAETITIA GiRAlDi ${ }^{2}$
}

\begin{abstract}
We study the effect of a rough wall on the controllability of micro-swimmers made of several balls linked by thin jacks: the so-called 3-sphere and 4-sphere swimmers. Our work completes the previous work [F. Alouges and L. Giraldi, Acta Applicandae Mathematicae 128 (2013) 153-179] dedicated to the effect of a flat wall. We show that a controllable swimmer (the 4-sphere swimmer) is not impacted by the roughness. On the contrary, we show that the roughness changes the dynamics of the 3-sphere swimmer, so that it can reach any direction almost everywhere.
\end{abstract}

Mathematics Subject Classification. 93B05.

Received September 30, 2013. Revised April 8, 2014.

Published online May 13, 2015.

\section{INTRODUCTION}

Micro-swimming is a subject of growing interest, notably for its biological and medical implications: one can mention the understanding of reproduction processes, the description of infection mechanisms, or the conception of micro-propellers for drug delivery in the body. As regards its mathematical modeling and analysis, the studies by Taylor [28], Lighthill [17] and Purcell [23] have been pioneering contributions to a constantly increasing field: we refer to the recent work of Powers and Lauga [15] for an extensive bibliography.

Among the many aspects of micro-swimming, the influence of the environment on swimmers dynamics has been recognized by many biological studies (see for instance $[5,22,25-27,31,32]$ ). One important factor in this dynamics is the presence of confining walls. For example, experiments have shown that some microorganisms, like E. Coli, are attracted to surfaces.

The focus of this paper is the effect of wall roughness on micro-swimming. Such effect has been already recognized in the context of microfluidics, in connection with superhydrophobic surfaces [16,33]. Moreover, recent studies have highlighted the role of roughness in the dynamics of passive spherical particles in a Stokes flow: we refer for instance to the study of Rad and Najafi [24] or to the one of Gérard-Varet and Hillairet [10].

We want here to study the impact of a rough wall on the displacement of micro-swimmers, at low Reynolds number. Our point of view will be theoretical, more precisely based on control theory. Connection between swimming at low Reynolds number and control theory has been emphasized over the last years

\footnotetext{
Keywords and phrases. Low-Reynolds number swimming, self-propulsion, three-sphere swimmer, rough wall effect, Lie brackets, control theory, asymptotic expansion.

* Supported by Direction Générale de l'Armement (DGA).

1 Institut de Mathématiques de Jussieu et Université Paris 7, Bâtiment Sophie Germain, 75205 Paris cedex 13, France.

2 Unité de Mathématiques Appliqueés (UMA), École Nationale Supérieure de Techniques Avancées (ENSTA-Paristech), 828

Boulevard des Marchaux, 91762 Palaiseau, France. laetitia.giraldi@polytechnique.edu.
} 
(see $[3,7,11,18-20])$. We shall ponder here on the recent studies $[2,4]$, dedicated to the controllability analysis of particular Stokesian robots, in the whole space and in the presence of a plane wall respectively. We shall here incorporate roughness at the wall, and focus on two classical models of swimmers: the 3-sphere swimmer (see $[1,2,4,12])$ and the 4 -sphere swimmer (see $[2,4])$. First, we will show that the controllability of the 4-sphere swimmer (already true near a flat wall) persists with roughness. Then, we will prove that the rough wall leads the 3 -sphere swimmer to reach any space direction. The underlying mechanism is the symmetry-breaking generated by the roughness.

The paper is divided into three parts. In Section 2, we introduce the mathematical model for the fluidswimmer coupling, and we derive from there an ODE for the swimmer dynamics. In Section 3, we show that the force field in this ODE is analytic with respect to the roughness amplitude and swimmer size and position. Combining this property with the results of [2] yields the controllability of the 4-sphere swimmer "almost everywhere" Section 4.1 provides an asymptotic expansion of the Dirichlet-to-Neumann operator, with respect to the roughness amplitude and swimmer's size. This operator is naturally involved in the expansion of the force fields. Eventually, we use this expansion and make it truly explicit in Section 4, in the special case of the 3 -sphere swimmer. This allows us to show its controllability.

\section{Mathematical Setting}

In this part, we present our mathematical model for the swimming problem.

\subsection{Swimmers}

We carry on the study of specific swimmers that were considered in [4] in $\mathbf{R}^{3}$ and in [2] in an half plane. These swimmers consist of $N$ spheres $\cup_{l=1}^{N} B_{l}$ of radii $a$ connected by $k$ thin jacks which are supposed free of viscous resistance. The position of the swimmer is described by a variable $\mathbf{p} \in \mathbf{R}^{3} \times S O(3)$, which gives both the coordinates of one point over the swimmer and the swimmer's orientation. Moreover, the shape variable is denoted by a $k$-tuple $\boldsymbol{\xi}$ : its $i$ th component $\xi_{i}$ gives the length of $i$ th arm, that can stretch or elongate through time. Nevertheless, the directions of the arms are only modified by global rotation of the swimmer. We call the set of the admissible state of swimmer $\mathcal{M}:=\{(\boldsymbol{\xi}, \mathbf{p})\}$. Let us stress that all the variables above depend implicitly on time, through the transport and deformation of the swimmer.

Many results of our paper apply to the general class of swimmers just described. Nevertheless, we will pay a special attention to two examples:

- The 4-sphere swimmer. We consider a regular tetrahedron $\left(\mathbf{S}_{1}, \mathbf{S}_{2}, \mathbf{S}_{3}, \mathbf{S}_{4}\right)$ with center $\mathbf{O} \in \mathbf{R}_{+}^{3}$. The 4-sphere swimmer consists of four balls linked by four arms of fixed directions $\overrightarrow{\mathbf{O S}}_{i}$ which are able to elongate and shrink (in a referential associated to the swimmer). The four ball cluster is completely described by the list of parameters $(\boldsymbol{\xi}, \mathbf{p})=\left(\xi_{1}, \ldots, \xi_{4}, \mathbf{x}_{c}, \boldsymbol{R}\right) \in(0, \infty)^{4} \times \mathbf{R}^{3} \times S O(3)$. Thus, the set of the admissible states of the swimmer is $\mathcal{M}=(0, \infty)^{4} \times \mathbf{R}^{3} \times S O(3)$. It is known that the 4-sphere swimmer is controllable in $\mathbf{R}^{3}$ and remains controllable in presence of a plane wall (see $[2,4]$ ). This means that it is able to move to any point and with any orientation under the constraint of being self-propelled, when the surrounding flow is dominated by viscosity (Stokes flow). This swimmer is depicted in Figure 1.

- The 3-sphere swimmer (see $[1,2,4]$ and $[21]$ ). It is composed of three aligned spheres, linked by two arms, see Figure 2. The dynamics of the swimmer is described through the lengths of the two arms $\xi_{1}, \xi_{2}$, the coordinates of the center of the middle ball: $\mathbf{x}_{c}=\left(x_{c}, y_{c}, z_{c}\right)$, and some matrix $\mathcal{R} \in S O(3)$ describing the orientation of the swimmer. Thus,

$$
(\boldsymbol{\xi}, \mathbf{p})=\left(\xi_{1}, \xi_{2}, \mathbf{x}_{c}, \mathcal{R}\right) \in(0, \infty)^{2} \times \mathbb{R}^{3} \times S O(3) .
$$

Here, the set of the admissible states for the 3 -sphere swimmer is $\mathcal{M}=(0, \infty)^{2} \times \mathbf{R}^{3} \times S O(3)$. As regards the position and elongation of the swimmer, the angle of the rotation $\mathcal{R}$ around the symmetry axis of the 3 -sphere is irrelevant. As a matter of fact, we will not show controllability for this angle: our result, Theorem 2.4, yields 


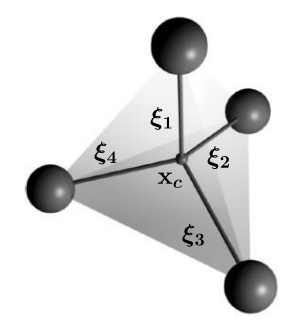

FiguRE 1. The four-sphere swimmer.

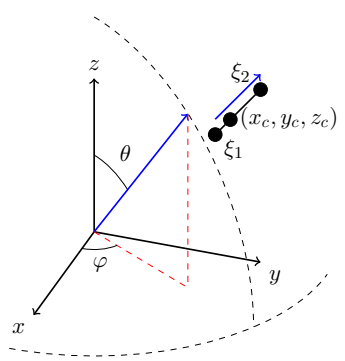

Figure 2. Coordinates of the 3-sphere swimmer.

controllability of the swimmer up to rotation around its axis. Still, the associated angular velocity is not zero, and will appear in the dynamics.

\subsection{Fluid flow}

We consider a fluid confined by a rough boundary. This boundary is modelled by a surface with equation $z=\varepsilon h(x, y)$, for some Lipschitz positive function $h$. Here, $\varepsilon>0$ denotes the amplitude of the roughness, that is $\|h\|_{\infty}=1$. The swimmer evolves in the half-space $\mathcal{O}=\left\{(x, y, z) \in \mathbf{R}^{3}\right.$ s. t. $\left.z>\varepsilon h(x, y)\right\}$. The fluid domain is then $\mathcal{F}:=\mathcal{O} \backslash \cup_{l=1}^{N} B_{l}$, and again it depends implicitly on time. Finally, we assume that the flow is governed there by the Stokes equation. Thus, the velocity $\mathbf{u}^{S}$ and the pressure $p^{S}$ of the fluid satisfy:

$$
-\mu \Delta \mathbf{u}^{S}+\nabla p^{S}=0, \operatorname{div} \mathbf{u}^{S}=0 \text { in } \mathcal{F},
$$

where $\mu$ is the viscosity of the fluid. We complement the Stokes equation (2.1) by standard no-slip boundary conditions, that read:

$$
\left\{\begin{array}{l}
\mathbf{u}^{S}=\Omega \times\left(\mathbf{x}-\mathbf{x}_{c}\right)+\mathbf{v}+\mathbf{u}_{d} \quad \text { at } \cup_{l=1}^{N} \partial B_{l}, \\
\mathbf{u}^{S}=0 \quad \text { at } \partial \mathcal{O} .
\end{array}\right.
$$

In other words, we impose the continuity of the velocity both at the fixed wall and at the boundary of the moving swimmer. Note that the velocity field of the swimmer is made of two parts:

- one corresponding to an (unknown) rigid movement, with angular velocity $\Omega$ and linear velocity $\mathbf{v}$. If $\mathbf{x}_{c}$ is the point over the swimmer encoded in $\mathbf{p}$, the velocity $\mathbf{v}$ is its speed. The vector $(\Omega, \mathbf{v})^{t}$ can be identified with $\dot{\mathbf{p}}$ (everything will be made explicit in due course).

- one corresponding to the (known) deformation of the jacks, with associated velocity $\mathbf{u}^{d}$, depending on $\dot{\boldsymbol{\xi}}$.

Introducing the Hilbert space

$$
\mathcal{V}=\left\{\mathbf{u} \in \mathcal{D}^{\prime}\left(\mathcal{F}, \mathbf{R}^{3}\right) \mid \nabla \mathbf{u} \in L^{2}(\mathcal{F}), \frac{\mathbf{u}(\mathbf{r})}{\sqrt{1+|\mathbf{r}|^{2}}} \in L^{2}(\mathcal{F})\right\},
$$

we get (for any configuration of the swimmer $\cup B_{l}$ and velocities $\left.\left(\Omega, \mathbf{v}, \mathbf{u}^{d}\right)\right)$ a unique solution $\left(\mathbf{u}^{S}, p^{S}\right)$ of $(2.1)$ $(2.2)$ in $\mathcal{V} \times L^{2}(\mathcal{F})$. See Appendix A for more details. 


\subsection{Dynamics}

Of course, the previous relations describe the equilibrium of the fluid flow at any given instant $t$. To close the model (that is the fluid-swimmer coupling), we still need to specify the dynamics of the swimmer, based on Newton's laws. The description is by now classical (see for instance $[4,18]$ ), and can be expressed by an affine control system without drift. Let us recall the principle of derivation. Neglecting inertia, Newton's laws become

$$
\left\{\begin{array}{l}
\sum_{l=1}^{N} \int_{\partial B_{l}} \boldsymbol{\sigma}\left(\mathbf{u}^{S}, p^{S}\right) \cdot \mathbf{n} \mathrm{d} s=0 \\
\sum_{l=1}^{N} \int_{\partial B_{l}} \boldsymbol{\sigma}\left(\mathbf{u}^{S}, p^{S}\right) \cdot \mathbf{n} \times\left(\mathbf{x}-\mathbf{x}_{c}\right) \mathrm{d} s=0
\end{array}\right.
$$

where $\boldsymbol{\sigma}(\mathbf{u}, p)=\mu\left(\nabla \mathbf{u}+\nabla^{t} \mathbf{u}\right)-p \mathbf{I} \mathbf{d}$ is the Cauchy tensor.

Moreover, if we introduce an orthonormal basis $\left(\mathbf{e}_{1}, \mathbf{e}_{2}, \mathbf{e}_{3}\right)$ and use linearity, $\mathbf{u}^{S}$ decomposes into

$$
\mathbf{u}^{S}=\sum_{i=1}^{3} \Omega_{i} \mathbf{u}_{i}+\sum_{i=4}^{6} v_{i-3} \mathbf{u}_{i}+\mathbf{u}^{d} .
$$

Here, the $\mathbf{u}_{i}$ 's and $\mathbf{u}^{d}$ are solutions of the Stokes equation, with zero Dirichlet condition at the wall, and inhomogeneous Dirichlet conditions at the ball. The Dirichlet data is $\mathbf{e}_{i} \times\left(\mathbf{x}-\mathbf{x}_{c}\right)$ for $i=1,2,3, \mathbf{e}_{i-3}$ for $i=4,5,6, \mathbf{u}_{d}$ for $\mathbf{u}^{d}$. Note also that the speed $\mathbf{u}^{d}$ can be expressed as a linear combination of $\left(\dot{\xi}_{i}\right)_{i=1}^{k}$ :

$$
\mathbf{u}^{d}=\sum_{i=1}^{k} \mathbf{u}_{i}^{d} \dot{\xi}_{i}
$$

Identifying $(\Omega, \mathbf{v})^{t}$ with $\dot{\mathbf{p}}$ (everything will be made explicit in due course), the system (2.4) reduces to the following ODE:

$$
\mathbf{M}^{a, \varepsilon}(\boldsymbol{\xi}, \mathbf{p}) \dot{\mathbf{p}}+\mathbf{N}^{a, \varepsilon}(\boldsymbol{\xi}, \mathbf{p})=0
$$

where the matrix $\mathbf{M}^{a, \varepsilon}(\boldsymbol{\xi}, \mathbf{p})$ is defined by,

$$
\mathbf{M}_{i, j}^{a, \varepsilon}(\boldsymbol{\xi}, \mathbf{p}):=\left\{\begin{array}{l}
\sum_{l=1}^{N} \int_{\partial B_{l}}\left(\left(\mathbf{x}-\mathbf{x}_{c}\right) \times \mathbf{e}_{i}\right) \cdot \boldsymbol{\sigma}\left(\mathbf{u}_{j}, p_{j}\right) \mathbf{n} \mathrm{d} s \quad(1 \leq i \leq 3,1 \leq j \leq 6), \\
\sum_{l=1}^{N} \int_{\partial B_{l}} \mathbf{e}_{i-3} \cdot \boldsymbol{\sigma}\left(\mathbf{u}_{j}, p_{j}\right) \mathbf{n} \mathrm{d} s \quad(4 \leq i \leq 6,1 \leq j \leq 6),
\end{array}\right.
$$

and $\mathbf{N}^{a, \varepsilon}(\boldsymbol{\xi}, \mathbf{p})$ is the vector of $\mathbb{R}^{6}$ whose entries are,

$$
\mathbf{N}_{i}^{a, \varepsilon}(\boldsymbol{\xi}, \mathbf{p}):=\left\{\begin{array}{l}
\sum_{l=1}^{N} \int_{\partial B_{l}}\left(\left(\mathbf{x}-\mathbf{x}_{c}\right) \times \mathbf{e}_{i}\right) \cdot \boldsymbol{\sigma}\left(\mathbf{u}^{d}, p^{d}\right) \mathbf{n} \mathrm{d} s \quad(1 \leq i \leq 3), \\
\sum_{l=1}^{N} \int_{\partial B_{l}} \mathbf{e}_{i-3} \cdot \boldsymbol{\sigma}\left(\mathbf{u}^{d}, p^{d}\right) \mathbf{n} \mathrm{d} s \quad(4 \leq i \leq 6) .
\end{array}\right.
$$

Of course, the matrix field $\mathbf{M}^{a, \varepsilon}$ and vector field $\mathbf{N}^{a, \varepsilon}$ depend on $a$, the radius of the sphere, and $\varepsilon$ the amplitude of the roughness at the wall. The matrix $\mathbf{M}^{a, \varepsilon}(\boldsymbol{\xi}, \mathbf{p})$ is checked to be symmetric and negative definite. By inverting it in (2.7), we end up with the following relation for the swimmer's dynamics:

$$
\dot{\mathbf{p}}=-\left(\mathbf{M}^{a, \varepsilon}(\boldsymbol{\xi}, \mathbf{p})\right)^{-1} \mathbf{N}^{a, \varepsilon}(\boldsymbol{\xi}, \mathbf{p}) .
$$


By using (2.6), we deduce that there is a family vector fields $\left(\mathbf{F}_{i}^{a, \varepsilon}\right)_{i=1}^{k}$ (each of them is defined on $\mathcal{M}$ and depends on the parameters $\epsilon$ the roughness of the wall and $a$ the radius of the sphere) such that equation (2.8) reads

$$
\dot{\mathbf{p}}=\sum_{i=1}^{k} \mathbf{F}_{i}^{a, \varepsilon}(\boldsymbol{\xi}, \mathbf{p}) \dot{\xi}_{i}
$$

We can also write the swimmer's dynamics (2.9) as:

$$
\left(\begin{array}{l}
\dot{\boldsymbol{\xi}} \\
\mathbf{p}
\end{array}\right)=\sum_{i=1}^{k} \mathbf{G}_{i}^{a, \varepsilon}(\boldsymbol{\xi}, \mathbf{p}) \dot{\xi}_{i}
$$

where $\mathbf{G}_{i}^{a, \varepsilon}:=\left(\begin{array}{c}\mathbf{e}_{i} \\ \mathbf{F}_{i}^{a, \varepsilon}\end{array}\right)\left(\left(\mathbf{e}_{1}, \ldots, \mathbf{e}_{k}\right)\right.$ is the canonical basis of $\left.\mathbb{R}^{k}\right)$.

Our goal will be to show local controllability of the ordinary differential system (2.10). We will fix an initial state of the swimmer, say $\left(\boldsymbol{\xi}^{i}, \mathbf{p}^{i}\right)$, and a final state not too far from the initial one, say $\left(\boldsymbol{\xi}^{f}, \mathbf{p}^{f}\right)$. Then, we will prove the following: for any final time $T>0$, there is a deformation function $t \mapsto \boldsymbol{\xi}(t)$ for which system (2.10) has a solution satisfying $(\boldsymbol{\xi}, \mathbf{p})(t=0)=\left(\boldsymbol{\xi}^{i}, \mathbf{p}^{i}\right),(\boldsymbol{\xi}, \mathbf{p})(t=T)=\left(\boldsymbol{\xi}^{f}, \mathbf{p}^{f}\right)$.

To make it rigorous, there will be two restrictions. First, we shall consider only admissible initial states: the spheres of the swimmer will neither overlap, nor touch the rough wall. Second, besides this natural assumption, we will need a more technical one. Namely, we will be able to show local controllability only for almost every $\left(a, \varepsilon, \boldsymbol{\xi}^{i}, \mathbf{p}^{i}\right)$. The expression almost every means here that the property will hold for $\left(a, \varepsilon, \boldsymbol{\xi}^{i}, \mathbf{p}^{i}\right)$ outside the set of zeros of a (non-trivial) analytic function. We refer to Definition 2.2 below for a precise meaning. Zeros of analytic functions may form complicated sets, but these sets are somehow small. For instance, analytic functions of one real variable have a countable number of zeroes. Hence, our result can be considered as a generic controllability result. Detailed statements are presented in the following section.

\subsection{Main results}

Before turning to our mathematical analysis, we synthetize here our main results.

The controllability properties of the swimmers will follow from a careful study of the properties of the $\mathbf{F}_{i}^{a, \varepsilon}$, s in (2.9). As a first consequence of this study, we will obtain the analyticity of these vector fields with respect to all parameters: the typical height of the roughness $\varepsilon$, the radius of the balls $a$, the vector of arms lengths $\boldsymbol{\xi}$ and the position of the swimmer $p$. More precisely, defining

$$
\mathcal{A}:=\left\{(\varepsilon, a, \boldsymbol{\xi}, \mathbf{p}) \in \mathbb{R} \times \mathbb{R}_{+}^{*} \times\left(\mathbb{R}_{+}^{*}\right)^{k} \times\left(\mathbb{R}^{3} \times S O(3)\right)\right. \text { such that }
$$

$$
\left.B_{i} \cap B_{j}=\emptyset \forall i \neq j, \text { and } B_{i} \cap \partial \mathcal{O}=\emptyset \forall i\right\},
$$

we have the following

Theorem 2.1. For all $i=1 \ldots k$, the field $\mathbf{F}_{i}^{a, \varepsilon}(\boldsymbol{\xi}, p)$ is an analytic function of $(\varepsilon, a, \boldsymbol{\xi}, \mathbf{p})$ over $\mathcal{A}$.

Then, as a consequence of Theorem 2.1, we will prove that the roughness does not change the controllability of the 4-sphere swimmer. We restrict here to local controllability "almost everywhere": this terminology refers to the following

Definition 2.2 (Almost everywhere). We say that a property holds for almost every $(\varepsilon, a, \boldsymbol{\xi}, \mathbf{p})$ in $\mathcal{A}$ if it holds for all $(\varepsilon, a, \boldsymbol{\xi}, \mathbf{p})$ outside the zero set of a (non-trivial) analytic function over $\mathcal{A}$. 
We have

Theorem 2.3. The 4-sphere swimmer is controllable almost everywhere, in the following sense: for almost every $\left(\varepsilon, a, \boldsymbol{\xi}^{i}, \mathbf{p}^{i}\right)$, one has local controllability from the initial configuration $\left(\boldsymbol{\xi}^{i}, \mathbf{p}^{i}\right)$. This means that for any final configuration $\left(\boldsymbol{\xi}^{f}, \mathbf{p}^{f}\right)$ in a small enough neighborhood of $\left(\boldsymbol{\xi}^{i}, \mathbf{p}^{i}\right)$ and any final time $T>0$, there exists $\boldsymbol{\xi} \in \mathcal{W}^{1, \infty}((0, T))$ satisfying $\boldsymbol{\xi}(0)=\boldsymbol{\xi}^{i}$ and $\boldsymbol{\xi}(T)=\boldsymbol{\xi}^{f}$ and such that if the self-propelled swimmer starts in position $\mathbf{p}^{i}$ with the shape $\boldsymbol{\xi}^{i}$ at time $t=0$, it ends at position $\mathbf{p}^{f}$ and shape $\boldsymbol{\xi}^{f}$ at time $t=T$ by changing its shape along $\boldsymbol{\xi}(t)$.

In the last Section 4, we shall address the controllability of the 3 -sphere swimmer. In the case of a flat boundary, as shown in [2], symmetries constrain the swimmer to move in a plane. Also, it does not rotate around its own axis. As we will see, the roughness at the wall breaks (in general) such symmetries, allowing for local controllability almost everywhere. Let us point here a subtlety regarding our controllability result. To express the dynamics of the swimmer through equation (2.9), we have included in variable $\mathbf{p}$ (more precisely in its $S O(3)$ component) an angle describing rotation of the 3 -sphere around its own axis. We are not able to show controllability for this angle: we only show controllability for the other components of $\mathbf{p}$. Of course, this is not a problem with regards to the effective movement of the swimmer: this angle is indeed irrelevant with regards to the swimmer's orientation and position. The analysis of Section 4 leads to the

Theorem 2.4. There exists a surface $h \in C_{c}^{\infty}\left(\mathbb{R}^{2}\right)$ such that the 3-sphere swimmer is locally controllable almost everywhere (up to rotation around its axis).

Refined statements will be provided in Section 4. This controllability result requires a careful asymptotic asymptotic expansion of the force fields $\mathbf{F}_{i}^{a, \varepsilon}$. This expansion is related to an expansion of a Dirichlet-to-Neumann map, performed in Section 4.1. Eventually, the dimension of the Lie algebra generated by the force fields is computed, and the controllability result follows from application of Chow's theorem (see Appendix B for more details on Chow's theorem).

\section{AnAlyticity OF THE DYNAMiCS}

\subsection{Regularity}

This paragraph is devoted to Theorem 2.1. We shall establish the analyticity of the vector fields $\mathbf{F}_{i}^{a, \varepsilon}$ with respect to $(\varepsilon, a, \boldsymbol{\xi}, \mathbf{p}) \in \mathcal{A}$. From the expressions (2.8) and (2.9), it comes down to proving the analyticity of the matrix function $\mathbf{M}$ and vector function $\mathbf{N}$ with respect to $(\varepsilon, a, \boldsymbol{\xi}, \mathbf{p})$ (note that $\mathbf{M} \mapsto \mathbf{M}^{-1}$ preserves analytic regularity). Thus, we fix an arbitrary $\overline{\mathbf{Y}}=(\bar{\varepsilon}, \bar{a}, \overline{\boldsymbol{\xi}}, \overline{\mathbf{p}}) \in \mathcal{A}$ and intend to show analyticity for $\mathbf{Y}=(\varepsilon, a, \boldsymbol{\xi}, \mathbf{p})$ near $\overline{\mathbf{Y}}$. The definitions of coefficients $\mathbf{M}_{i, j}$ and $\mathbf{N}_{i}$ are given prior to (2.8) and (2.9).

Let $\left(\mathbf{u}_{l}\right)_{1 \leqslant l \leqslant N}$ a family of $\mathrm{N}$ rigid vector fields, where every field in the family belongs to the "elementary set" $\left\{\mathbf{e}_{i} \times \mathbf{x}, \mathbf{e}_{i}, i=1 \ldots 3\right\}$. To such an "elementary family" $\left(\mathbf{u}_{l}\right)_{1 \leqslant l \leqslant N}$, we associate the solution $(\mathbf{u}, p)$ of the Stokes system in $\mathcal{F}$ with Dirichlet conditions:

$$
\mathbf{u}=0 \quad \text { at } \partial \mathcal{O}, \quad \mathbf{u}=\mathbf{u}_{l} \quad \text { at } \partial B_{l}, \quad l=1, \ldots, N .
$$

By linearity of the equations and boundary conditions, the Stokes solutions $\mathbf{u}_{i}(i=1 \ldots 6)$ and $\mathbf{u}^{d}$ introduced in paragraph 2.3 can be written as linear combinations of such "elementary Stokes solutions". One can then use this decomposition in the expressions for coefficients $\mathbf{M}_{i j}$ and $\mathbf{N}_{i}$. From there, to prove analyticity of these coefficients amounts to proving analyticity (in $\mathbf{Y}$ ) of the matrix functions

$$
\mathbf{I}:=\sum_{l=1}^{N} \int_{\partial B_{l}}\left(\begin{array}{l}
1 \\
\mathbf{x}
\end{array}\right) \otimes(\sigma(\mathbf{u}, p) n) d s \quad\left(\text { we recall } \mathbf{f} \otimes \mathbf{g}=\left(\mathbf{f}_{k} \mathbf{g}_{k^{\prime}}\right)\right)
$$

where $(\mathbf{u}, p)$ is any arbitrary elementary Stokes solution. 
To simplify a little the expression for $\mathbf{I}$, we first perform a change of variable. We denote by $\mathbf{x}_{l}$, resp. $\overline{\mathbf{x}}_{l}$ the center of the ball $B_{l}$, resp. the center of the ball $\bar{B}_{l}$. We introduce the affine functions

$$
\varphi_{l}(\mathbf{x}):=\frac{a}{\bar{a}}\left(\mathbf{x}-\overline{\mathbf{x}}_{l}\right)+\mathbf{x}_{l}, \quad 1 \leqslant l \leqslant N .
$$

Each $\varphi_{l}$ is a diffeomorphim from $\mathbb{R}^{3}$ to $\mathbb{R}^{3}$, sending $\bar{B}_{l}$ to $B_{l}$. This basic change of variable leads to

$$
I^{l}:=\int_{\partial B_{l}}\left(\begin{array}{l}
1 \\
\mathbf{x}
\end{array}\right) \otimes \sigma(\mathbf{u}, p) n \mathrm{~d} s=\frac{a}{\bar{a}} \int_{\partial \bar{B}_{l}}\left(\begin{array}{c}
1 \\
\varphi_{l}(\mathbf{x})
\end{array}\right) \otimes \sigma\left(\frac{\bar{a}}{a} \mathbf{u} \circ \varphi_{l}, p \circ \varphi_{l}\right) n \mathrm{~d} s
$$

(we recall $\left.\sigma(\mathbf{v}, q):=\nabla \mathbf{v}+(\nabla \mathbf{v})^{t}-q I d\right)$. The point in this change of variable is that the integral at the right-hand side is now over the fixed domain $\partial \bar{B}_{l}$. Then, we remark that $\left(\begin{array}{c}1 \\ \varphi_{l}(\mathbf{x})\end{array}\right)$ is analytic as a function of $\mathbf{Y}$ with values in $H^{1 / 2}\left(\partial \bar{B}_{l}\right)$. Actually, it is analytic with values in any reasonable space function: indeed, $\varphi_{l}$ is simply linear in $\left(a, \mathbf{x}_{l}\right)$. Thus, if we prove that $\sigma\left(\frac{\bar{a}}{a} \mathbf{u} \circ \varphi_{l}, p \circ \varphi_{l}\right) n$ is analytic as a function of $\mathbf{Y}$ with values in $H^{-1 / 2}\left(\partial \bar{B}_{l}\right)$, we will be able to conclude. Indeed, each coefficient $I_{k k^{\prime}}^{l}$ of the matrix function $I^{l}$ reads

$$
I_{k k^{\prime}}^{l}=\left\langle\left(\begin{array}{c}
1 \\
\varphi_{l}(\mathbf{x})
\end{array}\right)_{k}\left(\sigma\left(\frac{\bar{a}}{a} \mathbf{u} \circ \varphi_{l}, p \circ \varphi_{l}\right) n\right)_{k^{\prime}}\right\rangle_{\left\langle H^{1 / 2}, H^{-1 / 2}\right\rangle}
$$

and will therefore be analytic in $\mathbf{Y}$.

Last step is to establish analyticity of $\sigma\left(\frac{\bar{a}}{a} \mathbf{u} \circ \varphi_{l}, p \circ \varphi_{l}\right) n$, seen as a function of $\mathbf{Y}$ with values in $H^{-1 / 2}\left(\partial \bar{B}_{l}\right)$. Here, we recall a classical trace theorem, see ([29], Thm. 1.2) for a smooth open set $\Omega$, and for any divergencefree field $\mathbf{V} \in L^{2}(\Omega)$, we can define the generalized boundary value of its normal component $\mathbf{V} \cdot n \in H^{-1 / 2}(\partial \Omega)$. Moreover, the mapping

$$
L^{2}(\Omega) \rightarrow H^{-1 / 2}(\partial \Omega), \quad \mathbf{V} \mapsto \mathbf{V} \cdot n
$$

is continuous. Similar result holds for a divergence-free matrix $\mathbf{M}$ instead of $\mathbf{V}$, and $\mathbf{M} n$ instead of $\mathbf{V} \cdot n$ (looking separately at each line of the matrix). We can apply this last result with $\Omega:=\mathcal{F} \cap B\left(\overline{\mathbf{x}}_{l}, a+\eta\right)$ (the fluid part of a neighborhood of $\left.\overline{B_{l}}\right)$, and $\mathbf{M}:=\sigma\left(\frac{\bar{a}}{a} u \circ \varphi_{l}, p \circ \varphi_{l}\right)$. Indeed, $(\mathbf{u}, p)$ satisfies the homogeneous Stokes equation, which implies easily that $\mathbf{M}$ is divergence-free.

Hence, it is enough to show that for all $l=1 \ldots N$, for $\delta, \eta>0$ small enough:

$$
B(\overline{\mathbf{Y}}, \delta) \mapsto H^{1}\left(\mathcal{F} \cap B\left(\overline{\mathbf{x}}_{l}, a+\eta\right)\right) \times L^{2}\left(\mathcal{F} \cap B\left(\overline{\mathbf{x}}_{l}, a+\eta\right)\right), \quad \mathbf{Y} \mapsto\left(\mathbf{u} \circ \varphi_{l}, p \circ \varphi_{l}\right)
$$

is analytic. We could have replaced $B\left(\overline{\mathbf{x}}_{l}, a+\eta\right)$ by any neighborhood of $\partial \bar{B}_{l}$.

The problem is that $u$ is defined globally, as the solution of a Stokes system in $\mathcal{F}$. To benefit from this global information, we shall now construct a diffeomorphism $\varphi$ from $\mathbb{R}^{3}$ to $\mathbb{R}^{3}$, such that $\varphi=\varphi_{l}$ in a neighborhood of $\bar{B}_{l}$, for all $1 \leqslant l \leqslant N$. We will then prove analyticity in $\mathbf{Y}$ of the global fields $(\mathbf{u} \circ \varphi, p \circ \varphi)$. More precisely, we define

$$
\varphi(\mathbf{x}):=\mathbf{x}+\sum_{l} \chi\left(\mathbf{x}-\overline{\mathbf{x}}_{l}\right)\left(\varphi_{l}(\mathbf{x})-\mathbf{x}\right)+(\varepsilon-\bar{\varepsilon}) \chi_{h}(\mathbf{x})\left(0,0, h\left(x_{1}, x_{2}\right)\right)
$$

with $\chi, \chi_{h} \in C_{c}^{\infty}\left(\mathbb{R}^{3}\right), \chi=1$ near $B(0, \bar{a}), \chi_{h}=1$ near $x_{3}=\bar{\varepsilon} h\left(x_{1}, x_{2}\right)$. We take $\chi$ and $\chi_{h}$ with small enough supports, so that all $\chi\left(\cdot-\overline{\mathbf{x}}_{l}\right), 1 \leqslant l \leqslant N$ and $\chi_{h}$ have disjoint supports. With such choice, one has as expected $\varphi=\varphi_{l}$ near $\bar{B}_{l}$. In particular, $\varphi\left(\bar{B}_{l}\right)=B_{l}$. One has also correspondence of the rough boundaries: $\varphi(\partial \overline{\mathcal{O}})=\partial \mathcal{O}$. Moreover, for $\mathbf{Y} \in B(\overline{\mathbf{Y}}, \delta), \delta>0$ chosen small enough, it is easily seen that $\varphi$ can be written in the form

$$
\varphi(x)=x+\varphi_{1}(x)
$$

where $\varphi_{1}$ is a $C^{\infty}$ contractive function, say with $\left|\nabla \varphi_{1}\right| \leqslant \frac{1}{2}$ (the Lipschitz constant goes to zero with $\delta$ ). It follows that the differential of $\varphi$ is invertible at every point of $\mathbb{R}^{3}$, and that $\varphi$ is bijective from $\mathbb{R}^{3}$ to $\mathbb{R}^{3}$. 
This last statement is deduced from the following argument: for every fixed $y \in \mathbb{R}^{3}$, the equation $\varphi(x)=y$ can be written: $x=\varphi_{1, y}(x)$, where $\varphi_{1, y}(x)=y-\varphi_{1}(x)$. But $\varphi_{1, y}$ is contractive, so that by the Banach fixed point theorem, it has a unique fixed point $x$. Hence, $\varphi$ is bijective. Finally, the local inversion theorem together with the bijectivity of $\varphi$ show that $\varphi$ is a smooth diffeomorphism from $\mathbb{R}^{3}$ to $\mathbb{R}^{3}$. Taking onto account the image of the balls and the rough boundary: $\varphi(\overline{\mathcal{F}})=\mathcal{F}$. Also, it depends analytically on Y. Introducing $\mathbf{U}:=\mathbf{u} \circ \varphi$ and $P:=p \circ \varphi$, it remains to prove the following.

Claim: $\mathbf{Y} \mapsto(\mathbf{U}, P)$ is analytic from $B(\overline{\mathbf{Y}}, \delta)$ to $\overline{\mathcal{V}}_{0} \times L^{2}(\overline{\mathcal{F}})$, where

$$
\overline{\mathcal{V}}_{0}:=\left\{\mathbf{U} \in \mathcal{D}^{\prime}\left(\overline{\mathcal{F}}, \mathbf{R}^{3}\right)\left|\nabla \mathbf{U} \in L^{2}(\overline{\mathcal{F}}), \frac{\mathbf{U}(\mathbf{r})}{\sqrt{1+|\mathbf{r}|^{2}}} \in L^{2}(\overline{\mathcal{F}}), \quad \mathbf{U}\right|_{\partial \overline{\mathcal{O}}}=0\right\} .
$$

These spaces are standard ones for solvability of the Stokes equation. Note that fields in $\overline{\mathcal{V}}_{0}$ belong to $H_{l o c}^{1}$. By restriction of $\mathbf{U}$ and $P$ to a neighborhood of the balls $\bar{B}_{l}$, we will then obtain the expected analyticity on $\left(\mathbf{u} \circ \varphi_{l}, p \circ \varphi_{l}\right)$. To prove this claim, we shall use a classical path, namely relying on the implicit function theorem. Examples of such approach can be found in [13].

One first needs to write the system satisfied by $\mathbf{U}, P$. A simple computation yields

$$
\left\{\begin{aligned}
-\operatorname{div}(A \nabla \mathbf{U})+B \nabla P & =0 \quad \text { in } \overline{\mathcal{F}} \\
\operatorname{div}\left(B^{t} \mathbf{U}\right) & =0 \quad \text { in } \overline{\mathcal{F}} \\
\mathbf{U}=0 \quad \text { at } \partial \overline{\mathcal{O}}, \quad \mathbf{U} & =\mathbf{U}_{l} \quad \text { at } \partial \overline{\mathcal{B}}_{l}
\end{aligned}\right.
$$

where

$$
\begin{aligned}
& A=A(\mathbf{x}):=|\operatorname{det} \nabla \varphi(\mathbf{x})|\left(\nabla \varphi^{-1}\right)^{t}\left(\nabla \varphi^{-1}\right)(\varphi(\mathbf{x})), \\
& B=B(\mathbf{x}):=|\operatorname{det} \nabla \varphi(\mathbf{x})|\left(\nabla \varphi^{-1}\right)(\varphi(\mathbf{x})), \quad \mathbf{U}_{l}:=\mathbf{u}_{l} \circ \varphi_{l} .
\end{aligned}
$$

Note that $A, B, \mathbf{U}_{l}$ depend analytically on the parameter $\mathbf{Y}$. We now introduce

$$
\overline{\mathcal{V}}^{\prime}:=\text { the dual space of }\left\{\mathbf{U} \in \overline{\mathcal{V}}_{0},\left.\quad \mathbf{U}\right|_{\partial B_{l}}=0, \quad l=1 \ldots N\right\},
$$

and consider the mapping

$$
\begin{aligned}
& \mathcal{L}: \quad B(\overline{\mathbf{Y}}, \delta) \times \overline{\mathcal{V}}_{0} \times L^{2}(\overline{\mathcal{F}}) \mapsto \overline{\mathcal{V}}^{\prime} \times L^{2}(\overline{\mathcal{F}}) \times \prod_{l} H^{1 / 2}\left(\partial \bar{B}_{l}\right), \\
& (\mathbf{Y}, \mathbf{V}, Q) \mapsto\left(-\operatorname{div}(A \nabla \mathbf{V})+B \nabla Q, \operatorname{div}\left(B^{t} \mathbf{V}\right),\left(\left.\mathbf{V}\right|_{\partial \overline{\mathcal{B}}_{l}}-\mathbf{U}_{l}\right)_{l=1}^{N}\right)
\end{aligned}
$$

$\mathcal{L}$ is clearly well-defined, and it is analytic in $(\mathbf{Y}, \mathbf{V}, Q)$ : we refer to [30] for the definition of analytic functions over Banach spaces. Actually, the dependence of $\mathcal{L}$ in $(\mathbf{V}, Q)$ is elementary: $\mathcal{L}$ is an affine function in $(\mathbf{V}, Q)$. Moreover, $\mathbf{U}=\mathbf{U}_{\mathbf{Y}}$ and $P=P_{\mathbf{Y}}$ satisfy

$$
\mathcal{L}(\mathbf{Y}, \mathbf{U}, P)=0
$$

By the analytic version of the implicit function theorem, see again [30], $\mathbf{U}$ and $P$ will be analytic in $\mathbf{Y}$ near $\overline{\mathbf{Y}}$ if

$$
\left.\frac{\partial \mathcal{L}}{\partial(\mathbf{V}, Q)}\right|_{(\overline{\mathbf{Y}}, \mathbf{U}, P)} \text { is an isomorphism from } \overline{\mathcal{V}}_{0} \times L^{2}(\overline{\mathcal{F}}) \text { to } \overline{\mathcal{V}}^{\prime} \times L^{2}(\overline{\mathcal{F}}) \times \prod_{l} H^{1 / 2}\left(\partial \bar{B}_{l}\right)
$$

The computation of this partial differential is direct, taking into account that $\mathcal{L}$ is affine in $(\mathbf{V}, Q)$. It is invertible if and only if there is existence and uniqueness in $\overline{\mathcal{V}}_{0} \times L^{2}(\overline{\mathcal{F}})$ of a solution $(\mathbf{V}, Q)$ for the Stokes system

$$
\begin{aligned}
& -\Delta \mathbf{V}+\nabla Q=\mathbf{F} \quad \text { in } \overline{\mathcal{F}}, \\
& \operatorname{div} \mathbf{V}=G \quad \text { in } \overline{\mathcal{F}}, \\
& \mathbf{V}=\mathbf{V}_{l} \quad \text { at } \partial \bar{B}_{l}, \quad l=1 \ldots N
\end{aligned}
$$


where $\mathbf{F} \in \overline{\mathcal{V}}^{\prime}, G \in L^{2}(\overline{\mathcal{F}})$ and $\mathbf{V}_{l} \in H^{1 / 2}\left(\partial \bar{B}_{l}\right)$ are prescribed data. Note that the space $\overline{\mathcal{V}}_{0}$ encodes the additional boundary condition: $\mathbf{V}=0$ at $\partial \overline{\mathcal{O}}$.

The well-posedness of the previous system is established in the appendix. From there, we deduce the analyticity in $\mathbf{Y}$ of $(\mathbf{U}, P)$, which ends the proof of Theorem 2.1.

\subsection{Application to the 4-sphere swimmer}

From the analyticity shown above and the results of [2], we shall deduce Theorem 2.3. First, by replacing $k$ by 4 in (2.10), we write the equation of motion for the 4 -sphere swimmer as

$$
\left(\begin{array}{l}
\dot{\boldsymbol{\xi}} \\
\mathbf{p}
\end{array}\right)=\sum_{i=1}^{4} \mathbf{G}_{i}^{a, \varepsilon}(\boldsymbol{\xi}, \mathbf{p}) \dot{\xi}_{i} .
$$

For proving the local controllability of the swimmer, we can consider the Lie algebra generated by the family $\left(\mathbf{G}_{i}^{a, \varepsilon}\right)_{i=1}^{4}$. More precisely, by Chow's theorem (see Appendix B) one has local controllability at every $(\varepsilon, a, \boldsymbol{\xi}, \mathbf{p}) \in$ $\mathcal{A}$ satisfying

$$
\operatorname{Lie}_{(\boldsymbol{\xi}, \mathbf{p})}\left(\mathbf{G}_{1}^{a, \varepsilon}, \ldots, \mathbf{G}_{4}^{a, \varepsilon}\right)=T_{(\boldsymbol{\xi}, \mathbf{p})} \mathcal{M} .
$$

The algebra $\operatorname{Lie}_{(\boldsymbol{\xi}, \mathbf{p})}\left(\mathbf{G}_{1}^{a, \varepsilon}, \ldots, \mathbf{G}_{4}^{a, \varepsilon}\right)$ is said to be fully generated if the condition (3.4) is satisfied. Hence, Theorem 2.3 follows directly from

Lemma 3.1. For almost $(\varepsilon, a, \boldsymbol{\xi}, \mathbf{p}) \in \mathcal{A}$, the algebra Lie $(\boldsymbol{\xi}, \mathbf{p})\left(\mathbf{G}_{1}^{a, \varepsilon}, \ldots, \mathbf{G}_{4}^{a, \varepsilon}\right)$ associated to the 4-sphereswimmer (3.3) is equal to the tangent space $T_{(\boldsymbol{\xi}, \mathbf{p})} \mathcal{M}$.

Proof. First, we recall that the Lie algebra $\operatorname{Lie}_{(\boldsymbol{\xi}, \mathbf{p})}\left(\mathbf{G}_{1}^{a, \varepsilon}, \ldots, \mathbf{G}_{4}^{a, \varepsilon}\right)$ is always included in the tangent space $T_{(\boldsymbol{\xi}, \mathbf{p})} \mathcal{M}$. In particular, for every $(\varepsilon, a, \boldsymbol{\xi}, \mathbf{p}) \in \mathcal{A}$,

$$
10=T_{\mathbf{p}, \boldsymbol{\xi}} \mathcal{M} \geqslant \operatorname{dim} \operatorname{Lie}_{(\boldsymbol{\xi}, \mathbf{p})}\left(\mathbf{G}_{1}^{a, \varepsilon}, \ldots, \mathbf{G}_{4}^{a, \varepsilon}\right) .
$$

It is then enough to prove that for a.e. $(\varepsilon, a, \boldsymbol{\xi}, \mathbf{p}) \in \mathcal{A}, \operatorname{dim} \operatorname{Lie}_{(\boldsymbol{\xi}, \mathbf{p})}\left(\mathbf{G}_{1}^{a, \varepsilon}, \ldots, \mathbf{G}_{4}^{a, \varepsilon}\right) \geqslant 10$.

From [2], we know that the 4 -sphere swimmer is controllable in the flat case $\varepsilon=0$ : for almost every $(a, \boldsymbol{\xi}, \mathbf{p})$

$$
\operatorname{dim} \operatorname{Lie}_{(\boldsymbol{\xi}, \mathbf{p})}\left(\mathbf{G}_{1}^{a, 0}, \ldots, \mathbf{G}_{4}^{a, 0}\right)=10 .
$$

Hence, we can find 10 vector fields $\mathbf{G}_{i}=\mathbf{G}_{i}(\varepsilon, a, \boldsymbol{\xi}, \mathbf{p})$ in $\operatorname{Lie}_{(\boldsymbol{\xi}, \mathbf{p})}\left(\mathbf{G}_{1}^{a, \varepsilon}, \ldots, \mathbf{G}_{4}^{a, \varepsilon}\right)$ such that for almost every $(a, \boldsymbol{\xi}, \mathbf{p})$

$$
\operatorname{det}\left(\left(\mathbf{G}_{i}(0, a, \boldsymbol{\xi}, \mathbf{p})\right)_{i=1}^{10}\right) \neq 0 .
$$

Moreover, these vector fields, built upon Lie brackets of $\mathbf{G}_{1}^{a, \varepsilon}, \ldots, \mathbf{G}_{4}^{a, \varepsilon}$, can be chosen to be analytic in $(\varepsilon, a, \boldsymbol{\xi}, \mathbf{p})$. It follows that $\operatorname{dim} \operatorname{Lie}_{(\boldsymbol{\xi}, \mathbf{p})}\left(\mathbf{G}_{1}^{a, \varepsilon}, \ldots, \mathbf{G}_{4}^{a, \varepsilon}\right) \geqslant 10$ almost everywhere, namely outside the set of zeros of the analytic function

$$
\begin{aligned}
\operatorname{det}\left(\left(\mathbf{G}_{i}\right)_{i=1}^{10}\right): \mathbb{R} \times \mathbb{R} \times \mathcal{M} & \rightarrow \mathbb{R} \\
(\varepsilon, a, \boldsymbol{\xi}, \mathbf{p}) & \mapsto \operatorname{det}\left(\left(\mathbf{G}_{i}(\varepsilon, a, \boldsymbol{\xi}, \mathbf{p})\right)_{i=1}^{10}\right)
\end{aligned}
$$

which is non-trivial by (3.5).

\section{Controllability of the Three-Sphere swimmer}

The rest of the paper deals with the controllability of the 3-sphere swimmer, namely Theorem 2.4. As for the 4-sphere swimmer, the controllability result will derive from Chow's theorem, that is from a computation of the dimension of the Lie algebra of the family of the vector fields $\mathbf{G}_{i}^{a, \varepsilon}$ 's. The main difference with the case of the 4 -sphere swimmer is that controllability does not hold anymore when $\varepsilon=0$. Hence, one can not conclude by invoking a perturbation of the flat case. Also, a big difficulty comes from the fact that the vector fields $\mathbf{G}_{i}^{a, \varepsilon}$, $\mathrm{s}$ 
are not explicit and so not easily computable. To handle this difficulty, we shall express the vector fields in terms of the Dirichlet-to-Neumann operator, for which an accurate asymptotics in the regime of small roughness can be derived.

Let us outline here the steps of the proof of Theorem 2.4. First of all, in Section 4.1, we derive an asymptotic expansion of the Dirichlet-to-Neumann map of the Stokes problem, for small roughness $\varepsilon$ and small radius of the balls $a$. In Section 4.3, we deduce from it an asymptotic expansion of the dynamics (again for small roughness $\varepsilon$ and small radius of the balls a). From there, we can show in Section 4.4 that if a certain determinant function, defined in (4.50), is not trivial, then the Lie algebra of the $\mathbf{G}_{i}^{a, \varepsilon}$ 's is fully generated almost everywhere. The proof is completed by Appendix $\mathrm{C}$ where we ensure that this determinant function is not trivial (that is noneverywhere zero) for some roughness profile $h$.

\subsection{Asymptotic expansion of the Dirichlet-to-Neumann}

We now turn to the controllability properties of the 3-sphere swimmer. As mentioned before, the keypoint is to derive an asymptotic expansion of the Dirichlet-to-Neumann map of the Stokes operator, as parameters $a$ and $\varepsilon$ go to zero. Such expansion will be expressed in "the general case" where we consider $N$ balls in a fluid domain. Of course, in relation to Theorem 2.4, it will be used with $N=3$.

The reason for focusing on this map is that it appears in the definition of the fields $\mathbf{F}_{i}^{a, \varepsilon}$ : indeed, the definition of the coefficients $\mathbf{M}_{i j}^{a, \varepsilon}$ and $\mathbf{N}_{i}^{a, \varepsilon}$ involves

$$
D N: \prod_{l=1}^{N} H^{1 / 2}\left(\partial B_{l}\right) \mapsto \prod_{l=1}^{N} H^{-1 / 2}\left(\partial B_{l}\right), \quad\left(\mathbf{u}_{l}\right) \mapsto\left(\mathbf{f}_{l}:=\left.\sigma(\mathbf{u}, p) n\right|_{\partial B_{l}}\right),
$$

where $(\mathbf{u}, p)$ is the solution of the Stokes equation

$$
-\Delta \mathbf{u}+\nabla p=0, \quad \operatorname{div} \mathbf{u}=0 \quad \text { in } \mathcal{F},\left.\quad \mathbf{u}\right|_{\partial \mathcal{O}}=0,\left.\quad \mathbf{u}\right|_{\partial B_{l}}=\mathbf{u}_{l} .
$$

More precisely, it involves $D N$ in restriction to $N$-uplets of rigid vector fields over $B_{l}, l=1 \ldots N$. We denote by $R$ the (finite-dimensional) space of such $N$-uplets.

Even restricted to $R$, this operator is not very explicit: to derive directly an expansion in terms of the parameters of the swimmer and wall is not easy. Hence, we follow the same path as in $[2,4]$ : we write that for all $\left(\mathbf{u}_{l}\right)_{l=1}^{N} \in R$,

$$
D N\left(\left(\mathbf{u}_{l}\right)\right)=T^{-1}\left(\left(\mathbf{u}_{l}\right)\right)
$$

where

$$
T: \prod_{l=1}^{N} H^{-1 / 2}\left(\partial B_{l}\right) \mapsto \prod_{l=1}^{N} H^{1 / 2}\left(\partial B_{l}\right), \quad\left(\mathbf{f}_{l}\right) \mapsto\left(\mathbf{u}_{l}:=\left.\mathbf{u}\right|_{\partial B_{l}}\right)
$$

and $\mathbf{u}$ is the solution of the following Stokes system in $\mathcal{O}$ :

$$
-\Delta \mathbf{u}+\nabla p=\sum_{l=1}^{N} 1_{\partial B_{l}} \mathbf{f}_{l}, \quad \operatorname{div} \mathbf{u}=0 \quad \text { in } \quad \mathcal{O},\left.\quad \mathbf{u}\right|_{\partial \mathcal{O}}=0 .
$$

Equivalently, this last system can be written:

$$
-\Delta \mathbf{u}+\nabla p=0, \quad \operatorname{div} \mathbf{u}=0 \quad \text { in } \quad \mathcal{O} \backslash \cup_{l} \partial B_{l},\left.\quad[\mathbf{u}]\right|_{\partial B_{l}}=0,\left.\quad[\sigma(\mathbf{u}, p) n]\right|_{\partial B_{l}}=\mathbf{f}_{l},
$$

where $\left.[]\right|_{\partial B_{l}}$ denotes the jump across $\partial B_{l}$. Let us remind that $\mathcal{O}=\{z>\varepsilon h(x, y)\}$ is the domain without the balls. In particular, the operator $T$ (associated to a transmission condition) is not the Neumann-to-Dirichlet operator. The latter one would correspond to the Stokes problem

$$
-\Delta \mathbf{u}+\nabla p=0, \quad \operatorname{div} \mathbf{u}=0 \quad \text { in } \quad \mathcal{O} \backslash \cup_{l} B_{l},\left.\quad \sigma(\mathbf{u}, p) n\right|_{\partial B_{l}}=\mathbf{f}_{l},
$$


associated to a Neumann type condition. However, in restriction to the space $R$, the operators DN and $T^{-1}$ coincide, due to the fact that a rigid vector field is a solution of the Stokes equation, with zero pressure and zero stress tensor.

The advantage of $T$ over the Neumann-to-Dirichlet operator is its more explicit representation. Indeed, one has for all $i=1 \ldots N$

$$
T(\mathbf{f})_{i}(\mathbf{x})=\sum_{l=1}^{n} \int_{\partial B_{l}} \mathbf{K}^{\varepsilon}(\mathbf{x}, \mathbf{y}) \mathbf{f}_{l}(\mathbf{y}) \mathrm{d} \mathbf{y}, \quad \mathbf{x} \in \partial B_{i},
$$

where the kernel $\mathbf{K}^{\varepsilon}$ is simply the Green function associated to the Stokes equation in $\mathcal{O}$ : in other words, $\left(\mathbf{K}^{\epsilon}, \mathbf{q}^{\epsilon}\right)$ is the solution of the problem:

$$
\left\{\begin{aligned}
-\mu \Delta_{\mathbf{x}} \mathbf{K}^{\epsilon}\left(\mathbf{x}, \mathbf{x}_{0}\right)+\nabla_{\mathbf{x}} \mathbf{q}^{\epsilon}(\mathbf{x}) & =\delta_{\mathbf{x}_{0}}(\mathbf{x}) \mathbf{I}, \quad \mathbf{x} \text { in } \mathcal{O}, \\
\operatorname{div}_{\mathbf{x}} \mathbf{K}^{\varepsilon}\left(\mathbf{x}, \mathbf{x}_{0}\right) & =0, \quad \mathbf{x} \text { in } \mathcal{O} \\
\mathbf{K}^{\epsilon}\left(\mathbf{x}, \mathbf{x}_{0}\right) & =0 \quad \mathbf{x} \text { on } \partial \mathcal{O}
\end{aligned}\right.
$$

where I stands for the identity matrix. This will make easier the derivation of an asymptotic expansion, through an expansion of $T$. Still, there is one little technical difficulty: the domain of definition and range of $T$, that are $\prod_{l} H^{ \pm 1 / 2}\left(\partial B_{l}\right)$ depend on the parameter $a$ (and also on $(\mathbf{p}, \boldsymbol{\xi})$ ). Let us denote $B:=B(0,1)$ the unit ball, and $H_{N}^{ \pm 1 / 2}:=\left(H^{ \pm 1 / 2}(\partial B)\right)^{N}$. We introduce

$$
\varphi: \prod_{l=1}^{N} H^{1 / 2}\left(\partial B_{l}\right) \rightarrow H_{N}^{1 / 2}, \quad \mathbf{u}=\left(\mathbf{u}_{l}\right) \mapsto \mathbf{U}=\left(\mathbf{U}_{l}: \mathbf{r} \mapsto \mathbf{u}_{l}\left(\mathbf{x}_{l}+a \mathbf{r}\right)\right),
$$

as well as the adjoint map

$$
\varphi^{*}: H_{N}^{-1 / 2} \rightarrow \prod_{l=1}^{N} H^{-1 / 2}\left(\partial B_{l}\right), \quad \mathbf{F}=\left(\mathbf{F}_{l}\right) \mapsto \mathbf{f}=\left(\mathbf{f}_{l}\right),
$$

defined through the duality relation: $\left\langle\varphi^{*}(\mathbf{F}), \mathbf{u}\right\rangle=\langle\mathbf{F}, \varphi(\mathbf{u})\rangle$. Finally, we set $\mathcal{T}:=\varphi \circ T \circ \varphi^{*}: H_{N}^{-1 / 2} \mapsto H_{N}^{1 / 2}$. We shall use $\mathcal{T}$ rather than $T$ to compute the expansion of the force field in Section 4.3. Note that $\mathcal{T}$ depends implicitly on $\varepsilon, a$ and on $(\mathbf{p}, \boldsymbol{\xi})$. In what follows, we will always consider configurations in which the swimmer stays away from the rough wall:

$$
\operatorname{dist}\left(B_{l}, \partial \mathcal{O}\right) \geqslant \delta>0, \quad \forall l=1 \ldots N,
$$

for some given $\delta$.

\subsubsection{Expansion for small roughness $\varepsilon$}

Under the constraint (4.2), we prove

\section{Proposition 4.1.}

$$
\mathcal{T}:=\mathcal{T}^{0}+\varepsilon \mathcal{T}^{1}+O\left(\varepsilon^{2}\right) \quad \text { in } \quad \mathcal{L}\left(H_{N}^{-1 / 2}, H_{N}^{1 / 2}\right)
$$

where $\mathcal{T}^{0}$ and $\mathcal{T}^{1}$ are defined in (4.10) and (4.11)-(4.12) respectively.

Proof. For $\mathbf{f}=\left(\mathbf{f}_{l}\right) \in H_{N}^{-1 / 2}$, we can write

$$
\mathcal{T}(\mathbf{f})_{i}(r)=\sum_{j} \int_{\partial B} \mathbf{K}^{\varepsilon}\left(x_{i}+a \mathbf{r}, x_{j}+a \mathbf{s}\right) \mathbf{f}_{j}(\mathbf{s}) \mathrm{d} \mathbf{s}
$$

(with a classical and slightly abusive notation: the integral should be understood as a duality bracket). Thus, the whole point is to expand the kernel $\mathbf{K}^{\varepsilon}$ defined in (4.1). Of course, the first term should be $\mathbf{K}^{0}$, that is the 
Green function in the flat case. This Green function can be computed in terms of the Stokeslet by the method of images (see [6]): one has

$$
\mathbf{K}^{0}\left(\mathbf{r}, \mathbf{r}_{0}\right)=\mathbf{G}\left(\mathbf{r}-\mathbf{r}_{0}\right)+\mathbf{K}_{1}\left(\mathbf{r}, \mathbf{r}_{0}\right)+\mathbf{K}_{2}\left(\mathbf{r}, \mathbf{r}_{0}\right)+\mathbf{K}_{3}\left(\mathbf{r}, \mathbf{r}_{0}\right),
$$

the four functions $\mathbf{G}, \mathbf{K}_{1}, \mathbf{K}_{2}$ and $\mathbf{K}_{3}$ being respectively the Stokeslet

$$
\mathbf{G}(\mathbf{r})=\frac{1}{8 \pi \mu}\left(\frac{\mathbf{I d}}{|\mathbf{r}|}+\frac{\mathbf{r} \otimes \mathbf{r}}{|\mathbf{r}|^{3}}\right)
$$

and the three "images"

$$
\begin{gathered}
\mathbf{K}_{1}\left(\mathbf{r}, \mathbf{r}_{0}\right)=-\frac{1}{8 \pi \mu}\left(\frac{\mathbf{I d}}{\left|\mathbf{r}^{\prime}\right|}+\frac{\mathbf{r}^{\prime} \otimes \mathbf{r}^{\prime}}{\left|\mathbf{r}^{\prime}\right|^{3}}\right), \\
K_{2, i j}\left(\mathbf{r}, \mathbf{r}_{0}\right)=\frac{1}{4 \pi \mu} z_{0}^{2}\left(1-2 \delta_{j 3}\right)\left(\frac{\delta_{i j}}{\left|\mathbf{r}^{\prime}\right|^{3}}-\frac{3 r_{i}^{\prime} r_{j}^{\prime}}{\left|\mathbf{r}^{\prime}\right|^{5}}\right), \\
K_{3, i j}\left(\mathbf{r}, \mathbf{r}_{0}\right)=-\frac{1}{4 \pi \mu} z_{0}\left(1-2 \delta_{j 3}\right)\left(\frac{r_{3}^{\prime}}{\left|\mathbf{r}^{\prime}\right|^{3}} \delta_{i j}-\frac{r_{j}^{\prime}}{\left|\mathbf{r}^{\prime}\right|^{3}} \delta_{i 3}+\frac{r_{i}^{\prime}}{\left|\mathbf{r}^{\prime}\right|^{3}} \delta_{j 3}-\frac{3 r_{i}^{\prime} r_{j}^{\prime} r_{3}^{\prime}}{\left|\mathbf{r}^{\prime}\right|^{5}}\right) .
\end{gathered}
$$

Here $\mathbf{r}_{0}=\left(x_{0}, y_{0}, z_{0}\right)$ and $\mathbf{r}^{\prime}=\mathbf{r}-\tilde{\mathbf{r}}_{0}$, where $\tilde{\mathbf{r}}_{0}=\left(x_{0}, y_{0},-z_{0}\right)$ stands for the "image" of $\mathbf{r}_{0}$, that is to say, the point symmetric to $\mathbf{r}_{0}$ with respect to the flat wall.

We now consider $\mathbf{u}^{\varepsilon}\left(\mathbf{x}, \mathbf{x}_{0}\right)=\mathbf{K}^{\varepsilon}\left(\mathbf{x}, \mathbf{x}_{0}\right)-\mathbf{K}^{0}\left(\mathbf{x}, \mathbf{x}_{0}\right)$, for $\mathbf{x}_{0} \in \cup_{l} B_{l}$. As a function of $\mathbf{x}$, it satisfies the Stokes equation in $\mathcal{O}$ :

$$
-\Delta \mathbf{u}^{\varepsilon}\left(\cdot, \mathbf{x}_{0}\right)+\nabla \mathbf{p}\left(\cdot, \mathbf{x}_{0}\right)=0, \quad \operatorname{div} \mathbf{u}^{\varepsilon}\left(\cdot, \mathbf{x}_{0}\right)=0 \quad \text { in } \mathcal{O}
$$

with Dirichlet condition

$$
\mathbf{u}^{\varepsilon}\left(\cdot, \mathbf{x}_{0}\right)=-\mathbf{K}^{0}\left(\cdot, \mathbf{x}_{0}\right), \text { at } \partial \mathcal{O}
$$

We can then expand the boundary data: for $\mathbf{x}=(x, y, \varepsilon h(x, y)) \in \mathcal{O}$

$$
-\mathbf{K}^{0}\left(\mathbf{x}, \mathbf{x}_{0}\right)=-\sum_{k=1}^{n} \varepsilon^{k} \frac{h(x, y)^{k}}{k !} \partial_{z}^{k} \mathbf{K}^{0}\left(x, y, 0, \mathbf{x}_{0}\right)+O\left(\varepsilon^{n+1}\right) .
$$

More precisely, under the constraint (4.2), one has

$$
\left\|-\mathbf{K}^{0}\left(\cdot, x_{0}\right)+\sum_{k=1}^{n} \varepsilon^{k}\left(\mathbf{x} \mapsto \frac{h(x, y)^{k}}{k !} \partial_{z}^{k} \mathbf{K}^{0}\left(x, y, 0, \mathbf{x}_{0}\right)\right)\right\|_{H^{s}(\partial \mathcal{O})} \leqslant C_{\delta, s} \varepsilon^{n+1}, \quad \forall s .
$$

We deduce from this inequality that

$$
\left\|\nabla\left(\mathbf{u}^{\varepsilon}\left(\cdot, \mathbf{x}_{0}\right)-\sum_{k=1}^{n} \varepsilon^{k} \mathbf{u}^{k}\left(\cdot, \mathbf{x}_{0}\right)\right)\right\|_{L^{2}(\mathcal{O})} \leqslant C \varepsilon^{n+1}
$$

where $\mathbf{u}^{k}$ is the solution of

$$
\begin{aligned}
& -\Delta \mathbf{u}^{k}\left(\cdot, \mathbf{x}_{0}\right)+\nabla \mathbf{p}\left(\cdot, \mathbf{x}_{0}\right)=0, \quad \operatorname{div}^{k}\left(\cdot, \mathbf{x}_{0}\right)=0 \quad \text { in } \quad \mathcal{O} \\
& \mathbf{u}^{k}\left(\mathbf{x}, \mathbf{x}_{0}\right)=-\frac{h(x, y)^{k}}{k !} \partial_{z}^{k} \mathbf{K}^{0}\left(x, y, 0, \mathbf{x}_{0}\right), \quad \mathbf{x} \in \partial \mathcal{O} .
\end{aligned}
$$

The existence of the $\mathbf{u}^{k}$ 's and the estimate (4.8) are obtained by classical arguments (see the appendix for the more difficult case of a rough half-space minus the balls). In particular, we have

$$
\left\|\nabla\left(\mathbf{u}^{\varepsilon}\left(\cdot, \mathbf{x}_{0}\right)-\varepsilon \mathbf{u}^{1}\left(\cdot, \mathbf{x}_{0}\right)\right)\right\|_{L^{2}(\mathcal{O})} \leqslant C \varepsilon^{2} .
$$


The last step consists in replacing $\mathbf{u}^{1}$ by the solution $\mathbf{K}^{1}$ of

$$
\begin{aligned}
&-\Delta \mathbf{K}^{1}\left(\cdot, \mathbf{x}_{0}\right)+\nabla \mathbf{p}\left(\cdot, \mathbf{x}_{0}\right)=0, \quad \operatorname{div} \mathbf{K}^{1}\left(\cdot, \mathbf{x}_{0}\right)=0, \quad z>0, \\
& \mathbf{K}^{1}\left(x, y, 0, \mathbf{x}_{0}\right)=-h(x, y) \partial_{z} \mathbf{K}^{0}\left(x, y, 0, \mathbf{x}_{0}\right), \quad(x, y) \in \mathbb{R}^{2},
\end{aligned}
$$

that is replacing the rough half-space by the flat half-space. We claim that

$$
\left\|\nabla\left(\mathbf{u}^{1}\left(\cdot, \mathbf{x}_{0}\right)-\mathbf{K}^{1}\left(\cdot, \mathbf{x}_{0}\right)\right)\right\|_{L^{2}(\mathcal{O} \cap\{z>0\})}=O\left(\varepsilon^{2}\right) .
$$

With no loss of generality, we can assume that $h>0$ (meaning that the flat wall is below the rough wall). Otherwise, we can make an intermediate comparison with the solution $\tilde{\mathbf{K}}^{1}$ of the same Stokes problem in $\{z>-\varepsilon(\sup |h|+1)\}$. Now, an easy but important remark is that

$$
\left\|\mathbf{K}^{1}\left(\cdot, \mathbf{x}_{0}\right)\right\|_{H^{s}(\{0<z<Z\})} \leqslant C_{s, Z}, \quad \forall s \in \mathbb{N}, \forall Z>0 .
$$

Hence,

$$
\mathbf{K}^{1}\left(\mathbf{x}, \mathbf{x}_{0}\right)=-h(x, y) \partial_{z} \mathbf{K}^{0}\left(x, y, 0, \mathbf{x}_{0}\right)+O(\varepsilon) \text { in } H^{s}(\partial \mathcal{O}) .
$$

By a simple estimate on $\mathbf{u}^{1}-\mathbf{K}^{1}$, we deduce the claim.

Back to the definition of $\mathbf{u}^{\varepsilon}$, we obtain thanks to standard elliptic regularity in variable $\mathbf{x}$ : for all $\alpha \in \mathbb{N}^{3}$,

$$
\left|\partial_{\mathbf{x}}^{\alpha}\left(\mathbf{K}^{\varepsilon}\left(\mathbf{x}, \mathbf{x}_{0}\right)-\mathbf{K}^{0}\left(\mathbf{x}, \mathbf{x}_{0}\right)-\varepsilon \mathbf{K}^{1}\left(\mathbf{x}, \mathbf{x}_{0}\right)\right)\right|=O\left(\varepsilon^{2}\right),
$$

uniformly in $\mathbf{x}, \mathbf{x}_{0} \in \cup_{l} B_{l}$. The same reasoning as above can then be applied to the fields $\mathbf{u}_{\beta}^{\varepsilon}=\partial_{\mathbf{x}_{0}}^{\beta}\left(\mathbf{K}^{\varepsilon}-\mathbf{K}^{0}\right)$, for all $\beta \in \mathbb{N}^{3}$. Hence,

$$
\left|\partial_{\mathbf{x}}^{\alpha} \partial_{\mathbf{x}_{0}}^{\beta}\left(\mathbf{K}^{\varepsilon}\left(\mathbf{x}, \mathbf{x}_{0}\right)-\mathbf{K}^{0}\left(\mathbf{x}, \mathbf{x}_{0}\right)-\varepsilon \mathbf{K}^{1}\left(\mathbf{x}, \mathbf{x}_{0}\right)\right)\right|=O\left(\varepsilon^{2}\right),
$$

uniformly in $\mathbf{x}, \mathbf{x}_{0} \in \cup_{l} B_{l}$. The theorem follows straightforwardly, considering

$$
\mathcal{T}^{0}(\mathbf{f})_{i}(r):=\sum_{j} \int_{\partial B} \mathbf{K}^{0}\left(x_{i}+a \mathbf{r}, x_{j}+a \mathbf{s}\right) \mathbf{f}_{j}(\mathbf{s}) \mathrm{d} \mathbf{s}
$$

and

$$
\mathcal{T}^{1}(\mathbf{f})_{i}(r):=\sum_{j} \int_{\partial B} \mathbf{K}^{1}\left(x_{i}+a \mathbf{r}, x_{j}+a \mathbf{s}\right) \mathbf{f}_{j}(\mathbf{s}) \mathrm{d} \mathbf{s} .
$$

Expressing $\mathbf{K}^{1}\left(\mathbf{x}, \mathbf{x}_{0}\right)$ with a Poisson kernel yields

$$
\mathbf{K}^{1}\left(\mathbf{x}, \mathbf{x}_{0}\right):=-\int_{\partial \mathbb{R}_{+}^{3}} h(\mathbf{s}) \frac{\partial}{\partial z}\left(\mathbf{s} \mapsto \mathbf{K}^{0}(\mathbf{s}, \mathbf{x})\right) \frac{\partial}{\partial z}\left(\mathbf{s} \mapsto \mathbf{K}^{0}\left(\mathbf{s}, \mathbf{x}_{0}\right)\right) \mathrm{d} \mathbf{s} .
$$

where for simplicity we write $h(\mathbf{s})$ instead of $h\left(s_{1}, s_{2}\right)$, for $\mathbf{s}=\left(s_{1}, s_{2}, 0\right) \in \partial \mathbb{R}_{+}^{3}$.

\subsubsection{Expansion for small amplitude of spheres a}

We go one step further in the asymptotics of $\mathcal{T}$, by considering the regime of small radius $a$. The expression of $\mathcal{T}$ involves the maps

$$
\begin{aligned}
\mathcal{T}_{i, j}: H^{-1 / 2}(\partial B) & \rightarrow H^{1 / 2}(\partial B) \\
\mathbf{f}_{j} & \mapsto \int_{\partial B} \mathbf{K}\left(\mathbf{x}_{i}+a \cdot, \mathbf{x}_{j}+a \mathbf{s}\right) \mathbf{f}_{j}(\mathbf{s}) \mathrm{d} \mathbf{s},
\end{aligned}
$$

with the Green kernel K given by Proposition 4.1:

$$
\mathbf{K}\left(\mathbf{r}, \mathbf{r}^{\prime}\right):=\mathbf{G}\left(\mathbf{r}-\mathbf{r}^{\prime}\right)+\mathbf{K}_{1}\left(\mathbf{r}, \mathbf{r}^{\prime}\right)+\mathbf{K}_{2}\left(\mathbf{r}, \mathbf{r}^{\prime}\right)+\mathbf{K}_{3}\left(\mathbf{r}, \mathbf{r}^{\prime}\right)+\mathbf{K}_{4}\left(\mathbf{r}, \mathbf{r}^{\prime}\right) .
$$


We recall that $\mathbf{K}_{1}, \mathbf{K}_{2}$ and $\mathbf{K}_{3}$ are defined in (4.3), whereas $\mathbf{K}_{4}$ is defined by (see (4.12)):

$$
\mathbf{K}_{4}\left(\mathbf{r}, \mathbf{r}^{\prime}\right):=-\varepsilon \int_{\partial \mathbb{R}_{+}^{3}} h(\mathbf{s}) \frac{\partial}{\partial z}\left(\mathbf{s} \mapsto \mathbf{K}^{0}(\mathbf{s}, \mathbf{r})\right) \frac{\partial}{\partial z}\left(\mathbf{s} \mapsto \mathbf{K}^{0}\left(\mathbf{s}, \mathbf{r}^{\prime}\right)\right) \mathrm{d} \mathbf{s} .
$$

Eventually, we call $\mathcal{T}^{G}$ the Neumann to Dirichlet map associated to $\mathbf{G}$

$$
\begin{aligned}
\mathcal{T}^{G}: H^{-1 / 2}(\partial B) & \rightarrow H^{1 / 2}(\partial B) \\
\mathbf{f} & \mapsto \int_{\partial B} \mathbf{G}(a(\cdot-\mathbf{s})) \mathbf{f}(\mathbf{s}) \mathrm{d} \mathbf{s} .
\end{aligned}
$$

Proposition 4.2. Let $(i, j) \in\{1, \cdots, N\}^{2}$. We have the following expansions, valid for $a \ll 1$ :

- if $i \neq j$ then

$$
\mathcal{T}_{i, j}=\mathbf{K}\left(\mathbf{x}_{i}, \mathbf{x}_{j}\right)\left\langle\cdot, \mathbf{I}_{d}\right\rangle_{\partial B}+\mathbf{R}_{1}
$$

where $\left\|\mathbf{R}_{1}\right\|_{\mathcal{L}\left(H^{-1 / 2}, H^{1 / 2}\right)}=O(a)$,

- otherwise

$$
\mathcal{T}_{i, i}=\mathcal{T}^{G}+\sum_{k=1}^{4} \mathbf{K}_{k}\left(\mathbf{x}_{i}, \mathbf{x}_{i}\right)\left\langle\cdot, \mathbf{I}_{d}\right\rangle_{\partial B}+\mathbf{R}_{2}
$$

where $\left\|\mathbf{R}_{2}\right\|_{\mathcal{L}\left(H^{-1 / 2}, H^{1 / 2}\right)}=O(a)$.

Proof. Let $(i, j) \in\{1, \cdots, N\}^{2}$ be such that $i \neq j$. For all $\mathbf{f}_{j} \in H^{-1 / 2}(\partial B)$, we write

$$
\left(\mathcal{T}_{i, j}-\mathbf{K}\left(\mathbf{x}_{i}, \mathbf{x}_{j}\right)\left\langle\cdot, \mathbf{I}_{d}\right\rangle\right)\left(\mathbf{f}_{j}\right)(\mathbf{r})=\int_{\partial B}\left(\mathbf{K}\left(\mathbf{x}_{i}+a \mathbf{r}, \mathbf{x}_{j}+a \mathbf{s}\right)-\mathbf{K}\left(\mathbf{x}_{i}, \mathbf{x}_{j}\right)\right) \mathbf{f}_{j}(\mathbf{s}) \mathrm{d} \mathbf{s} .
$$

The point is that, as $i \neq j$, the kernel $\mathbf{K}$ is smooth in a neighborhood of $B_{i} \times B_{j}$. Hence,

$$
\left|\mathbf{K}\left(\mathbf{x}_{i}+a \mathbf{r}, \mathbf{x}_{j}+a \mathbf{s}\right)-\mathbf{K}\left(\mathbf{x}_{i}, \mathbf{x}_{j}\right)\right|=O(a),\left|\nabla \mathbf{K}\left(\mathbf{x}_{i}+a \mathbf{r}, \mathbf{x}_{j}+a \mathbf{s}\right)-\mathbf{K}\left(\mathbf{x}_{i}, \mathbf{x}_{j}\right)\right|=O(a)
$$

uniformly for $\mathbf{r}, \mathbf{s} \in B$. Estimate (4.14) follows straightforwardly.

The proof of (4.15) is similar: we have for all $\mathbf{f}_{i} \in H^{-1 / 2}(\partial B)$

$$
\left(\mathcal{I}_{i, i}-\mathcal{T}^{G}-\mathbf{K}\left(\mathbf{x}_{i}, \mathbf{x}_{j}\right)\left\langle\cdot, \mathbf{I}_{d}\right\rangle\right)\left(\mathbf{f}_{i}\right)(\mathbf{r})=\int_{\partial B} \sum_{k=1}^{4}\left(\mathbf{K}_{k}\left(\mathbf{x}_{i}+a \mathbf{r}, \mathbf{x}_{i}+a \mathbf{s}\right)-\mathbf{K}_{k}\left(\mathbf{x}_{i}, \mathbf{x}_{i}\right)\right) \mathbf{f}_{i}(\mathbf{s}) \mathrm{d} \mathbf{s},
$$

where none of the $\mathbf{K}_{k}$ 's is singular near $B_{i} \times B_{i}$.

As a simple consequence of the previous propositions, we have

Proposition 4.3. For every $\mathbf{f} \in H_{N}^{-1 / 2}$, for all $(\mathbf{x}, \boldsymbol{\xi})$,

$$
(\mathcal{T} \mathbf{f})_{i}(\mathbf{r})=\mathcal{T}^{G} \mathbf{f}_{i}+\sum_{l=1}^{4} \mathbf{K}_{l}\left(\mathbf{x}_{i}, \mathbf{x}_{i}\right)\left\langle\mathbf{f}_{i}, \mathbf{I d}\right\rangle_{\partial B}+\sum_{j \neq i} \mathbf{K}\left(\mathbf{x}_{i}, \mathbf{x}_{j}\right)\left\langle\mathbf{f}_{j}, \mathbf{I d}\right\rangle_{\partial B}+\mathcal{R}_{i}(\mathbf{f})
$$

with $\left\|\mathcal{R}_{i}\right\|_{\mathcal{L}\left(H_{N}^{-1 / 2}, H_{N}^{1 / 2}\right)}=O\left(a+\varepsilon^{2}\right)$, and $i=1 \ldots N$.

Proof. By Proposition 4.1: for all $i=1 \ldots N$, and all $\mathbf{r} \in \partial B$

$$
\begin{aligned}
(\mathcal{T} \mathbf{f})_{i}(\mathbf{r}) & :=\int_{\partial B} \mathbf{K}\left(\mathbf{x}_{i}+a \mathbf{r}, \mathbf{x}_{i}+a \mathbf{s}\right) \mathbf{f}_{i}(\mathbf{s}) \mathrm{d} s+\sum_{i \neq j} \int_{\partial B} \mathbf{K}\left(\mathbf{x}_{i}+a \mathbf{r}, \mathbf{x}_{j}+a \mathbf{s}\right) \mathbf{f}_{j}(\mathbf{s}) \mathrm{d} \mathbf{s}+\mathcal{R}^{\varepsilon}(\mathbf{f}) \\
& =\mathcal{T}_{i, i} \mathbf{f}_{i}+\sum_{j \neq i} \mathcal{T}_{i, j} \mathbf{f}_{j}+\mathcal{R}^{\varepsilon}(\mathbf{f}), \quad\left\|\mathcal{R}^{\varepsilon}\right\|_{\mathcal{L}\left(H_{N}^{-1 / 2}, H_{N}^{1 / 2}\right)}=O\left(\varepsilon^{2}\right)
\end{aligned}
$$

and the result follows from the application of (4.14) and (4.15) of Proposition 4.2. 
Proposition 4.4. For every $\mathbf{u} \in H_{N}^{1 / 2}$, for all (p, $\left.\boldsymbol{\xi}\right)$, one has

$$
\begin{aligned}
\left(\mathcal{T}^{-1} \mathbf{u}\right)_{i}= & \left(\mathcal{T}^{G}\right)^{-1}\left(\mathbf{u}_{i}-\sum_{k=1}^{4} \mathbf{K}_{k}\left(\mathbf{x}_{i}, \mathbf{x}_{i}\right)\left\langle\left(\mathcal{T}^{G}\right)^{-1} \mathbf{u}_{i}, \mathbf{I} \mathbf{d}\right\rangle_{\partial B}\right) \\
& -\left(\mathcal{T}^{G}\right)^{-1}\left(\sum_{j \neq i} \mathbf{K}\left(\mathbf{x}_{i}, \mathbf{x}_{j}\right)\left\langle\left(\mathcal{T}^{G}\right)^{-1} \mathbf{u}_{j}, \mathbf{I} \mathbf{d}\right\rangle_{\partial B}\right)+\tilde{\mathcal{R}}_{i}(\mathbf{u})
\end{aligned}
$$

with $\left\|\tilde{\mathcal{R}}_{i}\right\|_{\mathcal{L}\left(H_{N}^{1 / 2}, H_{N}^{-1 / 2}\right)}=O\left(a^{3}+a^{2} \varepsilon^{2}\right), i=1 \ldots N$.

Proof. We recall that

$$
\mathcal{T}^{G}: H^{-\frac{1}{2}}(\partial B) \rightarrow H^{\frac{1}{2}}(\partial B), \quad \mathbf{f} \mapsto \int_{\partial B} \mathbf{G}(a(\cdot-\mathbf{s})) \mathbf{f}(\mathbf{s}) \mathrm{d} \mathbf{s},
$$

and define for $l=1, \ldots, 4$ the operators

$$
\mathcal{S}_{l}: H^{-\frac{1}{2}}(\partial B) \rightarrow H^{\frac{1}{2}}(\partial B), \quad \mathbf{f} \mapsto \int_{\partial B} \mathbf{K}_{l}\left(\mathbf{x}_{i}, \mathbf{x}_{i}\right) \mathbf{f}(\mathbf{s}) \mathrm{d} \mathbf{s},
$$

and eventually

$$
\mathcal{S}_{i, j}: H^{-\frac{1}{2}}(\partial B) \rightarrow H^{\frac{1}{2}}(\partial B), \quad \mathbf{f} \mapsto \int_{\partial B} \mathbf{K}\left(\mathbf{x}_{i}, \mathbf{x}_{j}\right) \mathbf{f}(\mathbf{s}) \mathrm{d} \mathbf{s} .
$$

Notice that for all $\mathbf{f} \in H^{-\frac{1}{2}}(\partial B), \mathcal{S}_{l} \mathbf{f}$ and $\mathcal{S}_{i, j} \mathbf{f}$ are constant applications.

That these operators are continuous operators from $H^{-\frac{1}{2}}(\partial B)$ into $H^{\frac{1}{2}}(\partial B)$ is classical. We are only interested in estimating their norms, and more precisely in the way they depend on $a$ in the limit $a \rightarrow 0$. Notice that since the kernel $\mathbf{G}$ is homogeneous of degree -1 , one has

$$
\left\|\mathcal{T}^{G}\right\|_{\mathcal{L}\left(H^{-1 / 2}, H^{1 / 2}\right)}=O\left(\frac{1}{a}\right) \text { and }\left\|\left(\mathcal{T}^{G}\right)^{-1}\right\|_{\mathcal{L}\left(H^{1 / 2}, H^{-1 / 2}\right)}=O(a) .
$$

As far as $\mathcal{S}_{l}$ is concerned, we get that (since $\left|\mathbf{K}_{l}\left(\mathbf{x}_{i}, \mathbf{x}_{i}\right)\right|=O(1)$ )

$$
\left\|\mathcal{S}_{l}\right\|_{\mathcal{L}\left(H^{-1 / 2}, H^{1 / 2}\right)}=O(1)
$$

and similarly

$$
\left\|\mathcal{S}_{i, j}\right\|_{\mathcal{L}\left(H^{-1 / 2}, H^{1 / 2}\right)}=O(1) .
$$

When $a \rightarrow 0$ this enables us to invert (4.19) leading to (4.20).

\subsection{Remarks on the rotation of the swimmer around its own axis}

We must first come back to equations (2.7) and (2.9), in the particular case of the 3-sphere swimmer. Remember that the writing in this equation was slightly abusive: we had denoted by $\dot{\mathbf{p}}$ the vector $\left(\begin{array}{l}\Omega \\ \mathbf{v}\end{array}\right)$ associated to the rigid movement of the swimmer, see (2.5). In our case, $\Omega=\left(\begin{array}{c}\Omega_{1} \\ \Omega_{2} \\ \Omega_{3}\end{array}\right)$ and $\mathbf{v}=\dot{\mathbf{x}}_{c}=\left(\begin{array}{c}v_{1} \\ v_{2} \\ v_{3}\end{array}\right)$ are respectively the angular velocity and the linear velocity of the middle sphere, decomposed in an arbitrary orthonormal basis $\left(\mathbf{e}_{i}\right)$. Moreover, it is natural to take for $\mathbf{e}_{1}$ the unit vector of the 3 -sphere axis. Let $\theta$ be the angle between the swimmer's axis and $\mathbf{e}_{z}$, while $\varphi$ is the angle between the $x$-axis and the projection of the swimmer in $O x y$ plane (see Fig. 2). Then, the unit vector of the 3 -sphere axis reads (in the canonical basis) $\mathbf{e}_{1}=\left(\begin{array}{c}\cos (\varphi) \sin (\theta) \\ \sin (\varphi) \sin (\theta) \\ \cos (\theta)\end{array}\right)$. 
It is completed into an orthonormal basis by defining

$$
\mathbf{e}_{2}=\left(\begin{array}{c}
\cos (\varphi) \cos (\theta) \\
\sin (\varphi) \cos (\theta) \\
-\sin (\theta)
\end{array}\right), \quad \mathbf{e}_{3}=\left(\begin{array}{c}
-\sin (\varphi) \\
\cos (\varphi) \\
0
\end{array}\right)
$$

Hence, a rigorous writing of $(2.7)$ or $(2.9)$ is

$$
\mathbf{M}^{a, \varepsilon}\left(\begin{array}{c}
\Omega \\
\mathbf{v}
\end{array}\right)+\mathbf{N}^{a, \varepsilon}=0, \quad \text { or }\left(\begin{array}{c}
\Omega \\
\mathbf{v}
\end{array}\right)=-\left(\mathbf{M}^{a, \varepsilon}\right)^{-1} \mathbf{N}^{a, \varepsilon} .
$$

A crucial remark is that $\mathbf{M}^{a, \varepsilon}$ and $\mathbf{N}^{a, \varepsilon}$ do not depend on the whole of $\mathbf{p}$. The angle $\theta_{1}$ of rotation around the swimmer's axis is not involved, as it is irrelevant to the swimmer's position, orientation or elongation. In particular, keeping only the five bottom lines of the last system, we end up with a closed relation of the type

$$
\left(\begin{array}{c}
\dot{\theta}_{2} \\
\dot{\theta}_{3} \\
\dot{\mathbf{x}}_{c}
\end{array}\right)=\sum_{i=1}^{2} \tilde{\mathbf{F}}_{i}^{a, \varepsilon}\left(\theta_{2}, \theta_{3}, \mathbf{x}_{c}\right) \dot{\xi}_{i}
$$

where $\theta_{2}$ and $\theta_{3}$ are the rotation angles around $\mathbf{e}_{2}$ and $\mathbf{e}_{3}$ respectively. Then, by the analyticity of the $\tilde{\mathbf{F}}_{i}^{a, \varepsilon}$ and Chow's theorem, it remains to prove that there exists some $\left(\varepsilon, a, \theta_{2}, \theta_{3}, \mathbf{x}_{c}\right)$ such that

$$
\operatorname{dim} \operatorname{Lie}_{\left(\theta_{2}, \theta_{3}, \mathbf{x}_{c}\right)}\left(\left(\begin{array}{c}
1 \\
0 \\
\tilde{\mathbf{F}}_{1}
\end{array}\right),\left(\begin{array}{c}
0 \\
1 \\
\tilde{\mathbf{F}}_{2}
\end{array}\right)\right)=7 .
$$

Actually, we shall not work directly with angles $\theta_{2}, \theta_{3}$. We find it more convenient to work with the angles $\theta, \varphi$ introduced above (see Fig. 2). From the relation $\frac{d}{d t} \mathbf{e}_{1}=\Omega \times \mathbf{e}_{1}$, we infer that

$$
\Omega_{2}=-\sin \theta \dot{\varphi}, \quad \Omega_{3}=\dot{\theta} .
$$

Note that in the special case $\sin \theta=0$, the angle $\varphi$ coincides with the useless angle $\theta_{1}$. Moreover, the mapping $\left(\theta_{2}, \theta_{3}\right) \rightarrow(\theta, \varphi)$ is not a diffeomorphism in the vicinity of $\theta \equiv 0[\pi]$. Thus, we shall restrict to orientations of the swimmer for which

$$
|\sin \theta| \geqslant \delta>0
$$

We shall establish the maximality of the Lie algebra at points satisfying this condition.

Before entering the computation of this Lie algebra, we state a technical lemma, that will somehow allow us to neglect the rotation around the swimmer's axis. As mentioned before, we assume inequality (4.26). We have

Lemma 4.5. There exists a constant $C$ which does not depend on a and $\epsilon$ such that

$$
\left|\Omega_{1}\right| \leq C\left(|\dot{\theta}|+|\dot{\varphi}|+\left|\dot{\mathbf{x}}_{c}\right|+|\dot{\xi}|\right) .
$$

Proof. We go back to the first identity in (4.24). The first line gives

$$
\mathbf{M}_{1,1} \Omega_{1}=-\mathbf{N}_{1}+\mathbf{M}_{1,2} \sin (\theta) \dot{\varphi}-\mathbf{M}_{1,3} \dot{\theta}-\mathbf{M}_{1,4} v_{1}-\mathbf{M}_{1,5} v_{2}-\mathbf{M}_{1,6} v_{3} .
$$

We recall that, in the definitions of $\mathbf{M}$ and $\mathbf{N}$, we denoted by $\mathbf{u}_{i}$ and $\mathbf{u}^{d}$ some solutions of the Stokes equation, with zero Dirichlet condition at the wall, and inhomogeneous Dirichlet conditions at the ball. The Dirichlet data is $\mathbf{e}_{i} \times\left(\mathbf{x}-\mathbf{x}_{c}\right)$ for $i=1,2,3, \mathbf{e}_{i-3}$ for $i=4,5,6$, and $\mathbf{u}_{d}$ for $\mathbf{u}^{d}$. In the case of the 3 -sphere swimmer, $\mathbf{u}_{d}$ is $-\dot{\xi}_{1} \mathbf{e}_{1}$ on the sphere $\partial B_{1}, 0$ on the middle sphere and $\dot{\xi}_{2} \mathbf{e}_{1}$ on the sphere $\partial B_{3}$. 
Let us first examine

$$
\begin{aligned}
\mathbf{M}_{1,1} & =\sum_{l=1}^{3} \int_{\partial B}\left(\mathbf{x}_{l}-\mathbf{x}_{c}+a \mathbf{r}\right) \times \mathbf{e}_{1} \cdot\left(\mathcal{T}^{-1}\left(\mathbf{e}_{1} \times a \mathbf{r}, \mathbf{e}_{1} \times a \mathbf{r}, \mathbf{e}_{1} \times a \mathbf{r}\right)\right)_{l} \mathrm{~d} \sigma \\
& =3 \int_{\partial B} a \mathbf{r} \times \mathbf{e}_{1} \cdot\left(\mathcal{T}^{-1}\left(\mathbf{e}_{1} \times a \mathbf{r}, \mathbf{e}_{1} \times a \mathbf{r}, \mathbf{e}_{1} \times a \mathbf{r}\right)\right)_{l} \mathrm{~d} \sigma
\end{aligned}
$$

using that $\left(\mathbf{x}_{l}-\mathbf{x}_{c}\right) \times \mathbf{e}_{1}=0$. We then use the expansion (4.20). We recall the well-known fact that the rotation are eigenfunctions of $\left(\mathcal{T}^{G}\right)^{-1}$, with associated eigenvalue $3 \mu a$. In particular,

$$
\left(\mathcal{T}^{G}\right)^{-1}\left(\mathbf{e}_{1} \times a \mathbf{r}\right)=3 \mu a \mathbf{e}_{1} \times a \mathbf{r}, \quad \text { and }\left\langle\left(\mathcal{T}^{G}\right)^{-1}\left(\mathbf{e}_{1} \times a \mathbf{r}\right), \mathbf{I d}\right\rangle_{\partial B}=0 .
$$

We find then easily that $\mathbf{M}_{1,1}=-3 \mu a^{3}+O\left(a^{5}+\varepsilon^{2} a^{4}\right)$.

Then, we examine

$$
\mathbf{N}_{1}=\sum_{l=1}^{3} \int_{\partial B}\left(\mathbf{x}_{l}-\mathbf{x}_{c}+a \mathbf{r}\right) \times \mathbf{e}_{1} \cdot\left(\mathcal{T}^{-1}\left(-\dot{\xi}_{1} \mathbf{e}_{1}, 0, \dot{\xi}_{2} \mathbf{e}_{2}\right)\right)_{l} \mathrm{~d} \sigma
$$

Again, we can expand $\mathcal{T}^{-1}$ using (4.20). This time, we use that translations are eigenfunctions of $\left(\mathcal{T}^{G}\right)^{-1}$ with associated eigenvalue $\frac{3}{2} \mu a$. Thus,

$$
\left(\mathcal{T}^{G}\right)^{-1}\left(\mathbf{e}_{1}\right)=\frac{3}{2} \mu a \mathbf{e}_{1}
$$

It follows that the first terms in the expansion vanish, and we find

$$
\mathbf{N}_{1}=O\left(\left(a^{4}+a^{3} \varepsilon^{2}\right)|\dot{\boldsymbol{\xi}}|\right)
$$

The remaining terms $M_{1, j}, j=2, \ldots, 4$ can be handled with similar arguments. The lemma follows straightforwardly.

\subsection{Asymptotics of the 3-sphere dynamics}

We shall now provide an accurate description of the 3-sphere dynamics: broadly speaking, the point is to obtain an explicit expansion of the $\tilde{\mathbf{F}}_{i}$ 's in (4.25) (with angles $\theta_{2}, \theta_{3}$ replaced by $\theta, \varphi$, see remark above). We remind that the dynamics (that is the $6 \times 6$ system in (4.24)) is governed by self-propulsion: it corresponds to

- The sum of the forces on the swimmer being zero.

- The sum of the torques on the swimmer being zero.

Forces. By the definition of the swimmer, each sphere obeys a rigid body motion. More precisely, the velocity of each point $\mathbf{r}$ of the $l$ th sphere expresses as a sum of a translation and a rotation as

$$
\mathbf{u}_{l}^{S}(\mathbf{r})=\mathbf{u}_{T_{l}}+\mathbf{u}_{R_{l}}(\mathbf{r}),
$$

where $\mathbf{u}_{T_{l}}$ is constant on $\partial B$ while $\mathbf{u}_{R_{l}}(\mathbf{r})=\Omega \times a \mathbf{r}$ (remember that all quantities are expressed on the unit sphere $\partial B$ ). The vanishing of the total force, due to self-propulsion, reads

$$
\sum_{l} \int_{\partial B} \mathbf{f}_{l}=\sum_{l} \int_{\partial B}\left(\mathcal{T}^{-1}\left(\mathbf{u}_{1}^{S}, \mathbf{u}_{2}^{S}, \mathbf{u}_{3}^{S}\right)\right)_{l}=0 .
$$


Plugging (4.29) in (4.30) and using (4.20) leads to

$$
\begin{aligned}
\sum_{l} \int_{\partial B}\left(\mathcal{T}^{G}\right)^{-1} & \left(\mathbf{u}_{T l}+\mathbf{u}_{R l}-\sum_{k=1}^{4} \mathbf{K}_{k}\left(\mathbf{x}_{l}, \mathbf{x}_{l}\right)\left\langle\left(\mathcal{T}^{G}\right)^{-1}\left(\mathbf{u}_{T l}+\mathbf{u}_{R l}\right), \mathrm{Id}\right\rangle_{\partial B}\right) \\
& -\left(\mathcal{T}^{G}\right)^{-1}\left(\sum_{j \neq i} \mathbf{K}\left(\mathbf{x}_{i}, \mathbf{x}_{j}\right)\left\langle\left(\mathcal{T}^{G}\right)^{-1}\left(\mathbf{u}_{T l}+\mathbf{u}_{R l}\right), \mathbf{I d}\right\rangle_{\partial B}\right)=\left(O\left(a^{3}\right)+O\left(a^{2} \epsilon^{2}\right)\right)\|\mathbf{u}\|,
\end{aligned}
$$

where $\|\mathbf{u}\|=\left\|\left(\mathbf{u}_{i}^{S}\right)\right\|$ is any norm on the $n$-uplets of rigid vector fields over the ball.

Here,

$$
\|u\|=O\left(|\dot{\theta}|+|\dot{\varphi}|+\left|\dot{\mathbf{x}}_{c}\right|+\left|\Omega_{1}\right|\right)=O\left(|\dot{\theta}|+|\dot{\varphi}|+\left|\dot{\mathbf{x}}_{c}\right|\right)
$$

where the last equality comes from Lemma 4.5. As mentioned earlier, it is well known that both translations and rotations are eigenfunctions of the Dirichlet to Neumann map of the three dimensional Stokes operator outside a sphere. Namely

$$
\left(\mathcal{T}^{G}\right)^{-1} \mathbf{u}_{T l}=\lambda_{T} \mathbf{u}_{T l} \text { and }\left(\mathcal{T}^{G}\right)^{-1} \mathbf{u}_{R l}=\lambda_{R} \mathbf{u}_{R l} .
$$

It is also well-known that $\lambda_{T}=\frac{3 \mu a}{2}, \lambda_{R}=3 \mu a$, leading in particular to the celebrated Stokes formula

$$
\int_{\partial B}\left(\mathcal{T}^{G}\right)^{-1} \mathbf{u}_{T_{l}} \mathrm{~d} \mathbf{s}=6 \pi \mu a \mathbf{u}_{T_{l}}
$$

We also remark that due to $\int_{\partial B} \mathbf{u}_{R_{l}} \mathrm{~d} \mathbf{s}=0$, we have $\int_{\partial B}\left(\mathcal{T}^{G}\right)^{-1} \mathbf{u}_{R_{l}} \mathrm{~d} \mathbf{s}=0$. We therefore obtain

$$
6 \pi \mu a \sum_{l}\left(\mathbf{u}_{T l}-6 \pi \mu a \sum_{k=1}^{4} \mathbf{K}_{k}\left(\mathbf{x}_{l}, \mathbf{x}_{l}\right) \mathbf{u}_{T l}-6 \pi \mu a \sum_{j \neq i} \mathbf{K}\left(\mathbf{x}_{l}, \mathbf{x}_{j}\right) \mathbf{u}_{T j}\right)=\left(O\left(a^{3}\right)+O\left(a^{2} \epsilon^{2}\right)\right)\|\mathbf{u}\| .
$$

Torques. We now compute the torque with respect to the center $\mathbf{x}_{c}$ of the middle ball $B_{2}$. Self-propulsion of the swimmer implies that this torque vanishes:

$$
0=\int_{\partial B}\left(\mathbf{x}_{1}-\mathbf{x}_{2}+a \mathbf{r}\right) \times \mathbf{f}_{1}(\mathbf{r})+\int_{\partial B} a \mathbf{r} \times \mathbf{f}_{2}(\mathbf{r})+\int_{\partial B}\left(\mathbf{x}_{3}-\mathbf{x}_{2}+a \mathbf{r}\right) \times \mathbf{f}_{3}(\mathbf{r})=\mathbf{I}_{1}+\mathbf{I}_{2}+\mathbf{I}_{3},
$$

with the quantities $\mathbf{I}_{1}, \mathbf{I}_{2}$ and $\mathbf{I}_{3}$ given below.

$$
\begin{aligned}
\mathbf{I}_{1}= & \int_{\partial B}\left(\mathbf{x}_{1}-\mathbf{x}_{2}+a \mathbf{r}\right) \times \mathbf{f}_{1}(\mathbf{r})=\int_{\partial B}\left(-\xi_{1} \mathbf{e}_{1}+a \mathbf{r}\right) \times\left(\mathcal{T}^{-1}\left(\mathbf{u}_{1}^{S}, \mathbf{u}_{2}^{S}, \mathbf{u}_{3}^{S}\right)\right)_{1} \\
= & \int_{\partial B}\left(-\xi_{1} \mathbf{e}_{1}+a \mathbf{r}\right) \times\left(\mathcal{T}^{G}\right)^{-1}\left(\mathbf{u}_{T 1}+\mathbf{u}_{R 1}-6 \pi \mu a \sum_{k=1}^{4} \mathbf{K}_{k}\left(\mathbf{x}_{1}, \mathbf{x}_{1}\right) \mathbf{u}_{T 1}\right. \\
& \left.-6 \pi \mu a \sum_{j \neq 1} \mathbf{K}\left(\mathbf{x}_{1}, \mathbf{x}_{j}\right) \mathbf{u}_{T j}+O\left(a^{2}+a \varepsilon^{2}\right)\|\mathbf{u}\|\right) \\
= & -6 \pi \mu a \xi_{1} \mathbf{e}_{1} \times\left(\mathbf{u}_{T 1}-6 \pi \mu a \sum_{k=1}^{4} \mathbf{K}_{k}\left(\mathbf{x}_{1}, \mathbf{x}_{1}\right) \mathbf{u}_{T 1}-6 \pi \mu a \sum_{j \neq 1} \mathbf{K}\left(\mathbf{x}_{1}, \mathbf{x}_{j}\right) \mathbf{u}_{T j}\right) \\
& +\left(O\left(a^{3}\right)+O\left(a^{2} \epsilon^{2}\right)\right)\|\mathbf{u}\| .
\end{aligned}
$$


Similarly, we get,

$$
\begin{aligned}
\mathbf{I}_{2}= & a \int_{\partial B} \mathbf{r} \times \mathbf{f}_{2}(\mathbf{r})=a \int_{\partial B} \mathbf{r} \times\left(\mathcal{T}^{-1}\left(\mathbf{u}_{1}^{S}, \mathbf{u}_{2}^{S}, \mathbf{u}_{3}^{S}\right)\right)_{2} \\
= & a \int_{\partial B} \mathbf{r} \times\left(\mathcal{T}^{G}\right)^{-1}\left(\mathbf{u}_{T 2}+\mathbf{u}_{R 2}-6 \pi \mu a \sum_{k=1}^{4} \mathbf{K}_{k}\left(\mathbf{x}_{2}, \mathbf{x}_{2}\right) \mathbf{u}_{T 2}\right. \\
& \left.-6 \pi \mu a \sum_{j \neq 2} \mathbf{K}\left(\mathbf{x}_{2}, \mathbf{x}_{j}\right) \mathbf{u}_{T j}+O\left(a^{2}+a \varepsilon^{2}\right)\|\mathbf{u}\|\right) \\
= & O\left(a^{3}+a^{3} \epsilon^{2}\right)\|\mathbf{u}\| .
\end{aligned}
$$

Finally,

$$
\begin{aligned}
\mathbf{I}_{3}= & \int_{\partial B}\left(\mathbf{x}_{3}-\mathbf{x}_{2}+a \mathbf{r}\right) \times \mathbf{f}_{3}(\mathbf{r}) \\
= & 6 \pi \mu a \xi_{2} \mathbf{e}_{1} \times\left(\mathbf{u}_{T 3}-6 \pi \mu a \sum_{k=1}^{4} \mathbf{K}_{k}\left(\mathbf{x}_{3}, \mathbf{x}_{3}\right) \mathbf{u}_{T 3}-6 \pi \mu a \sum_{j \neq 3} \mathbf{K}\left(\mathbf{x}_{3}, \mathbf{x}_{j}\right) \mathbf{u}_{T j}\right) \\
& +\left(O\left(a^{3}\right)+O\left(a^{2} \epsilon^{2}\right)\right)\|\mathbf{u}\| .
\end{aligned}
$$

Denoting by $\mathbf{A}$ the matrix

$$
\mathbf{A}=\left(\begin{array}{lll}
\mathbf{A}_{11} & \mathbf{A}_{12} & \mathbf{A}_{13} \\
\mathbf{A}_{21} & \mathbf{A}_{22} & \mathbf{A}_{23} \\
\mathbf{A}_{31} & \mathbf{A}_{32} & \mathbf{A}_{33}
\end{array}\right)
$$

where for $i=1,2,3$

$$
\mathbf{A}_{i i}=\mathbf{I d}-6 \pi \mu a \sum_{l=1}^{4} \mathbf{K}_{l}\left(\mathbf{x}_{i}, \mathbf{x}_{i}\right)
$$

and for $i, j=1,2,3$ with $i \neq j$

$$
\mathbf{A}_{i j}=-6 \pi \mu a \mathbf{K}\left(\mathbf{x}_{i}, \mathbf{x}_{j}\right)
$$

and $\mathbf{S}$ the matrix

$$
\mathbf{S}=\left(\begin{array}{ccc}
\mathbf{I d} & \mathbf{I d} & \mathbf{I d} \\
-\xi_{1} \mathbf{e}_{1} \times & 0 & +\xi_{2} \mathbf{e}_{1} \times
\end{array}\right)
$$

we can rewrite the self-propulsion assumption (4.33) and (4.34) as

$$
\mathbf{S A}\left(\begin{array}{l}
\mathbf{u}_{T_{1}} \\
\mathbf{u}_{T_{2}} \\
\mathbf{u}_{T_{3}}
\end{array}\right)=\left(O\left(a^{2}\right)+O\left(a \epsilon^{2}\right)\right)\|\mathbf{u}\| .
$$

Terms involving the $\mathbf{u}_{R_{l}}$ 's are included in the r.h.s.

We now express $\mathbf{u}_{T_{1}}, \mathbf{u}_{T_{2}}$ and $\mathbf{u}_{T_{3}}$ in terms of $\dot{\mathbf{x}}_{c}, \dot{\theta}, \dot{\varphi}$ and $\dot{\boldsymbol{\xi}}$. Since $\mathbf{u}_{T_{2}}$ is the velocity of the center of the ball $B_{2}$, one has

$$
\mathbf{u}_{T_{2}}=\dot{\mathbf{x}}_{c}=\left(\begin{array}{c}
\dot{x} \\
\dot{y} \\
\dot{z}
\end{array}\right) \quad \text { in the canonical basis of } \mathbb{R}^{3} \text {. }
$$

Then, by using $\frac{d}{d t} \mathbf{e}_{1}=\dot{\theta} \mathbf{e}_{2}+\sin (\theta) \dot{\varphi} \mathbf{e}_{3}$, we get

$$
\mathbf{u}_{T_{1}}=\mathbf{u}_{T_{2}}-\xi_{1}\left(\dot{\theta} \mathbf{e}_{2}+\sin (\theta) \dot{\varphi} \mathbf{e}_{3}\right)-\dot{\xi}_{1} \mathbf{e}_{1}, \quad \mathbf{u}_{T_{3}}=\mathbf{u}_{T_{2}}+\xi_{2}\left(\dot{\theta} \mathbf{e}_{2}+\sin (\theta) \dot{\varphi} \mathbf{e}_{3}\right)+\dot{\xi}_{2} \mathbf{e}_{1}
$$


In matrix form, all this reads Then, the speed $\mathbf{u}_{T_{i}}(i=1,2,3)$ is expressed as

$$
\left(\begin{array}{c}
\mathbf{u}_{T_{1}} \\
\mathbf{u}_{T_{2}} \\
\mathbf{u}_{T_{3}}
\end{array}\right)=\mathbf{T}\left(\begin{array}{c}
\Omega_{1} \\
\dot{\theta} \\
\dot{\varphi} \\
\dot{x} \\
\dot{y} \\
\dot{z}
\end{array}\right)+\mathbf{U} \dot{\boldsymbol{\xi}}
$$

with

$$
\mathbf{T}=\left(\begin{array}{ccc}
0-\xi_{1} \mathbf{e}_{2} & -\xi_{1} \sin (\theta) \mathbf{e}_{3} & \mathbf{I d} \\
0 & 0 & \mathbf{I d} \\
0 & 0 & \mathbf{d} \\
0 & 0 & \\
0+\xi_{2} \mathbf{e}_{2}+\xi_{2} \sin (\theta) \mathbf{e}_{3} & \mathbf{I d}
\end{array}\right), \quad \text { and } \quad \mathbf{U}=\left(\begin{array}{cc}
-\mathbf{e}_{1} & 0 \\
0 & \vdots \\
\vdots & 0 \\
0 & \mathbf{e}_{1}
\end{array}\right)
$$

Combining with (4.38), the motion equation (4.24) becomes

$$
\left(\mathbf{S A}+\mathbf{R}_{1}\right)\left(\mathbf{T}\left(\begin{array}{c}
\Omega_{1} \\
\dot{\theta} \\
\dot{\varphi} \\
\dot{x} \\
\dot{y} \\
\dot{z}
\end{array}\right)+\left(\mathbf{U}+\mathbf{R}_{2}\right) \dot{\boldsymbol{\xi}}\right)=0
$$

where the residual matrices $\mathbf{R}_{1}, \mathbf{R}_{2}$ satisfy

$$
\left|\mathbf{R}_{1}\right|+\left|\mathbf{R}_{2}\right|=\left(O\left(a^{2}\right)+O\left(a \epsilon^{2}\right)\right)
$$

using (4.32). Finally, we only keep the five bottom lines of this system. It yields the following $5 \times 5$ system

$$
(\tilde{\mathbf{S}} \mathbf{A}+\tilde{\mathbf{R}})\left(\tilde{\mathbf{T}}\left(\begin{array}{c}
\dot{\theta} \\
\dot{\varphi} \\
\dot{x} \\
\dot{y} \\
\dot{z}
\end{array}\right)+\mathbf{U} \dot{\boldsymbol{\xi}}\right)=0
$$

where

$$
\tilde{\mathbf{S}}:=\left(S_{i, j}\right)_{2 \leqslant i \leqslant 6,1 \leqslant j \leqslant 9}, \quad \tilde{\mathbf{T}}:=\left(\begin{array}{ccc}
-\xi_{1} \mathbf{e}_{2}-\xi_{1} \sin (\theta) \mathbf{e}_{3} & \mathbf{I d} \\
0 & 0 & \\
0 & 0 & \mathbf{I d} \\
0 & 0 & \\
+\xi_{2} \mathbf{e}_{2}+\xi_{2} \sin (\theta) \mathbf{e}_{3} & \mathbf{I d}
\end{array}\right),
$$

and where the residual matrices still satisfy $\left|\tilde{\mathbf{R}}_{1}\right|+\left|\tilde{\mathbf{R}}_{2}\right|=O\left(a^{2}\right)+O\left(a \epsilon^{2}\right)$. We leave to the reader to check that $\tilde{\mathbf{S}} \mathbf{A} \tilde{\mathbf{T}}=\tilde{\mathbf{S}} \tilde{\mathbf{T}}+O(a)$ is invertible, with $\left|(\tilde{\mathbf{S}} \mathbf{A} \tilde{\mathbf{T}})^{-1}\right|=O(1)$ uniformly in $a$ and $\varepsilon$. Then, we can write system (4.41) as

$$
\left(\begin{array}{c}
\dot{\theta} \\
\dot{\varphi} \\
\dot{x} \\
\dot{y} \\
\dot{z}
\end{array}\right)=-(\tilde{\mathbf{S}} \mathbf{A} \tilde{\mathbf{T}})^{-1} \tilde{\mathbf{S}} \mathbf{A} \mathbf{U} \dot{\boldsymbol{\xi}}+\tilde{\mathbf{R}} \dot{\boldsymbol{\xi}}
$$

with $|\tilde{\mathbf{R}}|=O\left(a^{2}+\varepsilon^{2} a\right)$. 


\subsection{Reachable set}

We are now ready to prove Theorem 2.4. In all what follows, in order to lighten the notation, we drop the tilda, $\varepsilon$ and $a$ in the ODE (4.42) and express it as

$$
\dot{\mathbf{X}}=\mathbf{F}_{1}(\mathbf{X}) \dot{\xi}_{1}+\mathbf{F}_{2}(\mathbf{X}) \dot{\xi}_{2}, \quad \mathbf{X}:=\left(\begin{array}{c}
\xi_{1} \\
\xi_{2} \\
\theta \\
\varphi \\
x \\
y \\
z
\end{array}\right)
$$

To expand the $\mathbf{F}_{i}$ 's, we decompose the matrix $\mathbf{A}$ into three matrices: $\mathbf{A}:=\mathbf{I d}+\mathbf{A}^{1}+\mathbf{A}^{2}$ where

$$
\mathbf{A}_{i i}^{1}=-6 \pi \mu a \sum_{k=1}^{3} \mathbf{K}_{k}\left(\mathbf{x}_{i}, \mathbf{x}_{i}\right) \forall i \quad \mathbf{A}_{i j}^{1}=-6 \pi \mu a\left(\mathbf{G}\left(\mathbf{x}_{i}, \mathbf{x}_{j}\right)+\sum_{k=1}^{3} \mathbf{K}_{k}\left(\mathbf{x}_{i}, \mathbf{x}_{j}\right)\right) \forall i \neq j
$$

and where

$$
\mathbf{A}_{i, j}^{2}=-6 \pi \mu a \mathbf{K}_{4}\left(\mathbf{x}_{i}, \mathbf{x}_{j}\right) \quad \forall i, j
$$

Thanks to (4.41), we get an expansion of the form $\mathbf{F}_{i}:=\mathbf{F}_{i}^{0}+\mathbf{F}_{i}^{1}+\mathbf{F}_{i}^{2}+\mathbf{R}_{i}$ where $\mathbf{F}_{i}^{0}, \mathbf{F}_{i}^{1}$ and $\mathbf{F}_{i}^{2}$ are respectively the zero order term, the term of order $a$ and the term of order $\varepsilon a$. The remainder is $\mathbf{R}_{i}=\left(O\left(a^{2}\right)+O\left(a \epsilon^{2}\right)\right)$. These vector fields are given by

$$
\begin{aligned}
& \mathbf{F}_{i}^{0}=\left(\begin{array}{c}
\mathbf{e}_{i} \\
-(\mathbf{S T})^{-1}(\mathbf{S U}) \mathbf{e}_{i}
\end{array}\right), \\
& \mathbf{F}_{i}^{1}=\left(\begin{array}{cc}
\left((\mathbf{S T})^{-1} \mathbf{S A}^{1} \mathbf{T}(\mathbf{S T})^{-1} \mathbf{S U}-(\mathbf{S T})^{-1} \mathbf{S} \mathbf{A}^{1} \mathbf{U}\right) \mathbf{e}_{i}
\end{array}\right), \\
& \mathbf{F}_{i}^{2}=\left(\begin{array}{c}
0 \\
\left((\mathbf{S T})^{-1} \mathbf{S} \mathbf{A}^{2} \mathbf{T}(\mathbf{S T})^{-1} \mathbf{S U}-(\mathbf{S T})^{-1} \mathbf{S} \mathbf{A}^{2} \mathbf{U}\right) \mathbf{e}_{i}
\end{array}\right) .
\end{aligned}
$$

where $\mathbf{e}_{1}=\left(\begin{array}{l}1 \\ 0\end{array}\right)$ and $\mathbf{e}_{2}=\left(\begin{array}{l}0 \\ 1\end{array}\right)$.

Now, we want to find some $(\varepsilon, a, \mathbf{X})$ for which the determinant

$$
\operatorname{det}(\mathbf{X}):=\left|\mathbf{F}_{1}, \mathbf{F}_{2},\left[\mathbf{F}_{1}, \mathbf{F}_{2}\right],\left[\mathbf{F}_{1},\left[\mathbf{F}_{1}, \mathbf{F}_{2}\right]\right],\left[\mathbf{F}_{2},\left[\mathbf{F}_{1}, \mathbf{F}_{2}\right]\right],\left[\mathbf{F}_{1},\left[\mathbf{F}_{1},\left[\mathbf{F}_{1}, \mathbf{F}_{2}\right]\right]\right],\left[\mathbf{F}_{2},\left[\mathbf{F}_{2},\left[\mathbf{F}_{1}, \mathbf{F}_{2}\right]\right]\right]\right|(\mathbf{X}) \neq 0
$$

As the l.h.s. defines an analytic function of $\mathbf{X}$, it will be non-zero almost everywhere. Thus, the Lie algebra generated by $\mathbf{F}_{1}$ and $\mathbf{F}_{2}$ will be maximal (of dimension 7) at almost every $\mathbf{X}$, and local controllability will follow from Chow's theorem, see [14].

For all $\mathbf{G} \in \operatorname{Lie}\left(\mathbf{F}_{1}, \mathbf{F}_{2}\right)$, let us denote $\mathbf{G}^{0}, \mathbf{G}^{1}$ and $\mathbf{G}^{2}$ the zero order term, the term of order $a$ and the term of order $a \varepsilon$ in the expansion of the vector field $\mathbf{G}$ respectively. Thus,

$$
\mathbf{G}=\mathbf{G}^{0}+\mathbf{G}^{1}+\mathbf{G}^{2}+O\left(a^{2}\right)+O\left(a \epsilon^{2}\right) .
$$

For instance the expansion of the first Lie bracket reads

$$
\left[\mathbf{F}_{1}, \mathbf{F}_{2}\right]=\left[\mathbf{F}_{1}, \mathbf{F}_{2}\right]^{0}+\left[\mathbf{F}_{1}, \mathbf{F}_{2}\right]^{1}+\left[\mathbf{F}_{1}, \mathbf{F}_{2}\right]^{2}+O\left(a^{2}\right)+O\left(a \epsilon^{2}\right),
$$


with

$$
\left[\mathbf{F}_{1}, \mathbf{F}_{2}\right]^{0}=\left[\mathbf{F}_{1}^{0}, \mathbf{F}_{2}^{0}\right], \quad\left[\mathbf{F}_{1}, \mathbf{F}_{2}\right]^{1}=\left[\mathbf{F}_{1}^{1}, \mathbf{F}_{2}^{0}\right]+\left[\mathbf{F}_{1}^{0}, \mathbf{F}_{2}^{1}\right], \quad\left[\mathbf{F}_{1}, \mathbf{F}_{2}\right]^{2}=\left[\mathbf{F}_{1}^{2}, \mathbf{F}_{2}^{0}\right]+\left[\mathbf{F}_{1}^{0}, \mathbf{F}_{2}^{2}\right] .
$$

Note that for all $\mathbf{G} \in \operatorname{Lie}\left(\mathbf{F}_{1}, \mathbf{F}_{2}\right), \mathbf{G}^{0}+\mathbf{G}^{1}$ is a "flat wall" expansion, first order in $a$. Meanwhile, $\mathbf{G}^{2}$ is the first term which takes into account the roughness.

Without including this extra term, the three-sphere swimmer would not be controllable (see [2]), meaning that the determinant would vanish. We have notably

Lemma 4.6. For all $\mathbf{G} \in \operatorname{Lie}\left(\mathbf{F}_{1}, \mathbf{F}_{2}\right) \backslash\left\{\mathbf{F}_{1}, \mathbf{F}_{2}\right\}, \mathbf{G}^{0}=0$.

Proof. A simple calculation yields

$$
\mathbf{F}_{1}^{0}(\mathbf{X})=\left(\begin{array}{c}
1 \\
0 \\
0 \\
0 \\
\frac{1}{3} \cos (\varphi) \sin (\theta) \\
\frac{1}{3} \sin (\varphi) \sin (\theta) \\
\frac{1}{3} \cos (\theta)
\end{array}\right), \quad \mathbf{F}_{2}^{0}(\mathbf{X})=\left(\begin{array}{c}
0 \\
1 \\
0 \\
0 \\
-\frac{1}{3} \cos (\varphi) \sin (\theta) \\
-\frac{1}{3} \sin (\varphi) \sin (\theta) \\
-\frac{1}{3} \cos (\theta)
\end{array}\right) .
$$

It implies that $\left[\mathbf{F}_{1}^{0}, \mathbf{F}_{2}^{0}\right]$ is zero. The lemma is proved.

As regards the $O(a)$ term, we have

Lemma 4.7. Let Lie $\left(\mathbf{F}_{1}, \mathbf{F}_{2}\right)^{1}:=\left\{\mathbf{G}^{0}+\mathbf{G}^{1}\right.$ s.t. $\left.\mathbf{G} \in \operatorname{Lie}\left(\mathbf{F}_{1}, \mathbf{F}_{2}\right)\right\}$. For all $\mathbf{X}$, the dimension of the subspace Lie $\left(\mathbf{F}_{1}, \mathbf{F}_{2}\right)^{1}(\mathbf{X})$ is less than 5 .

Proof. As said above, for all $\mathbf{G} \in \operatorname{Lie}\left(\mathbf{F}_{1}, \mathbf{F}_{2}\right)$, the sum $\mathbf{G}^{0}+\mathbf{G}^{1}$ is a $O(a)$ expansion of the "flat wall field", corresponding to the case $h=0$. But in such flat case, symmetries constrain the swimmer within a plane. Thus, the associated manifold has at most dimension $5\left(\xi_{1}, \xi_{2}\right.$, two coordinates for the center of the middle ball, one angle). This implies the result.

Remark 4.8. Since without roughness the swimmer evolves in a plane, it follows that the angle $\varphi$ cannot change with time. Consequently, for all $\mathbf{F}(\mathbf{X}) \in \operatorname{Lie}\left(\mathbf{F}_{1}, \mathbf{F}_{2}\right)^{1}(\mathbf{X})$ the fourth component of the vector $\mathbf{F}(\mathbf{X})$ is zero.

Remark 4.9. The Lemma 4.7 also applies to the vector fields which do not take into account the roughness i.e., the ones which appear in the expansion without $\varepsilon$.

From this, we will get that the non-zero leading term in the expansion of det has power $a^{5} \epsilon^{2}$. Theorem 2.4 follows directly from

Proposition 4.10. In the regime $1 \gg \varepsilon \gg a$, one can find a surface $h \in C_{c}^{\infty}\left(\mathbb{R}^{2}\right)$ and a non-trivial analytic function $\mathcal{B}^{h}$ defined on $\mathcal{M}$ such that for all $\mathbf{X}$

$$
\operatorname{det}(\mathbf{X})=a^{5} \epsilon^{2} \mathcal{B}^{h}(\mathbf{X})+O\left(a^{6} \varepsilon^{2}+a^{5} \epsilon^{3}\right) .
$$


Proof. For all vector $\mathbf{G}$, we denote $(\mathbf{G})_{j^{\prime}}^{j}:=\left(\mathbf{G}_{k}\right)_{j \leqslant k \leqslant j^{\prime}}$. Since $\mathbf{F}_{i}, i=1,2$, is of the type $\left(\begin{array}{c}\mathbf{e}_{i} \\ * \\ \vdots \\ *\end{array}\right)$, we get easily that

$$
\operatorname{det}(\mathbf{X})=\left|\mathbf{Z}_{1}, \mathbf{Z}_{2}, \mathbf{Z}_{3}, \mathbf{Z}_{4}, \mathbf{Z}_{5}\right|
$$

where

$$
\left\{\begin{array}{l}
\mathbf{Z}_{1}:=\left(\left[\mathbf{F}_{1}, \mathbf{F}_{2}\right]\right)_{7}^{3} \\
\mathbf{Z}_{2}:=\left(\left[\mathbf{F}_{1},\left[\mathbf{F}_{1}, \mathbf{F}_{2}\right]\right]\right)_{7}^{3} \\
\mathbf{Z}_{3}:=\left(\left[\mathbf{F}_{2},\left[\mathbf{F}_{1}, \mathbf{F}_{2}\right]\right]\right)_{7}^{3} \\
\mathbf{Z}_{4}:=\left(\left[\mathbf{F}_{1},\left[\mathbf{F}_{1},\left[\mathbf{F}_{1}, \mathbf{F}_{2}\right]\right]\right]\right)_{7}^{3} \\
\mathbf{Z}_{5}:=\left(\left[\mathbf{F}_{2},\left[\mathbf{F}_{2},\left[\mathbf{F}_{1}, \mathbf{F}_{2}\right]\right]\right]\right)_{7}^{3}
\end{array}\right.
$$

From Lemma 4.6, $\mathbf{Z}_{i}^{0}=0$ for all $i=1 \ldots 5$. Moreover, by Lemma 4.7, any determinant of the type

$$
\left|\mathbf{Z}_{k_{1}}^{1}, \mathbf{Z}_{k_{2}}^{1}, \mathbf{Z}_{k_{3}}^{1}, \mathbf{Z}_{k_{4}}^{1}\right|, \quad k_{i} \in\{1, \ldots, 5\} \quad \text { is zero. }
$$

Expanding the function det by 5 -linearity, we obtain

$$
\operatorname{det}(\mathbf{X})=a^{5} \epsilon^{2} \mathcal{B}^{h}(\mathbf{X})+O\left(a^{6} \varepsilon^{2}+a^{5} \epsilon^{3}\right)
$$

where the function $\mathcal{B}^{h}(\mathbf{X})$ is defined as follows. Let

$$
\mathcal{I}:=\left\{\mathbf{k} \in\{1, \ldots, 5\}^{5} \quad \text { with } k_{1}<k_{2} \quad \text { and } \quad k_{3}<k_{4}<k_{5} \quad \text { distinct of } k_{1} \text { and } k_{2}\right\} .
$$

We set

$$
\mathcal{B}^{h}(\mathbf{X}):=\sum_{\mathbf{k} \in \mathcal{I}} \pm\left|\mathbf{Z}_{k_{1}}^{2}, \mathbf{Z}_{k_{2}}^{2}, \mathbf{Z}_{k_{3}}^{1}, \mathbf{Z}_{k_{4}}^{1}, \mathbf{Z}_{k_{5}}^{1}\right|
$$

where the \pm is the signature of the permutation $i \rightarrow k_{i}$.

It remains to prove that there exists $\mathbf{X}_{0}$ such that $\mathcal{B}\left(\mathbf{X}_{0}\right)$ is non-zero. By calling $\mathbf{K}_{4}^{\text {int }}$ the function $\left(\mathbf{s}, \mathbf{r}, \mathbf{r}^{\prime}\right) \mapsto$ $\frac{\partial}{\partial z}\left(\mathbf{s} \mapsto \mathbf{K}^{0}(\mathbf{s}, \mathbf{r})\right) \frac{\partial}{\partial z}\left(\mathbf{s} \mapsto \mathbf{K}^{0}\left(\mathbf{s}, \mathbf{r}^{\prime}\right)\right)$, we have (see $\left.(4.12)\right)$

$$
\mathbf{K}_{4}\left(\mathbf{r}, \mathbf{r}^{\prime}\right)=-\varepsilon \int_{\partial \mathbb{R}_{+}^{3}} h(\mathbf{s}) \mathbf{K}_{4}^{i n t}\left(\mathbf{s}, \mathbf{r}, \mathbf{r}^{\prime}\right) \mathrm{d} \mathbf{s} .
$$

We then define the $3 \times 3$ block matrix $\mathbf{A}_{\text {int }}^{2}(\mathbf{s})$ through

$$
\left(\mathbf{A}_{\text {int }}^{2}(\mathbf{s})\right)_{i j}=-6 \pi \mu a \mathbf{K}_{4}^{i n t}\left(\mathbf{s}, \mathbf{x}_{i}, \mathbf{x}_{j}\right), \quad i, j=1 \ldots 3 .
$$

By using the linearity of the integral, the vector fields $\mathbf{F}_{i}^{2}, i=1,2 \mathrm{read}$

$$
\mathbf{F}_{i}^{2}=\left(-\varepsilon \int_{\partial \mathbb{R}_{+}^{3}} h(\mathbf{s})\left(\mathbf{F}_{i, i n t}^{2}(\mathbf{s})\right) \mathrm{d} \mathbf{s}\right),
$$

where,

$$
\mathbf{F}_{i, i n t}^{2}(\mathbf{s})=-\left(-(\mathbf{S T})^{-1} \mathbf{S} \mathbf{A}_{\text {int }}^{2}(\mathbf{s}) \mathbf{T}(\mathbf{S T})^{-1} \mathbf{S U}+(\mathbf{S T})^{-1} \mathbf{S} \mathbf{A}_{\text {int }}^{2}(\mathbf{s}) \mathbf{U}\right) \mathbf{e}_{i}
$$

Then, denoting

$$
\mathbf{Z}_{1, \text { int }}^{2}(\mathbf{s}):=\left[\mathbf{F}_{1, \text { int }}^{2}(\mathbf{s}), \mathbf{F}_{2}^{0}\right]+\left[\mathbf{F}_{1}^{0}, \mathbf{F}_{2, \text { int }}^{2}(\mathbf{s})\right]
$$


leads to

$$
\mathbf{Z}_{1}^{2}=-\varepsilon \int_{\partial \mathbb{R}_{+}^{3}} h(\mathbf{s}) \mathbf{Z}_{1, \text { int }}^{2}(\mathbf{s}) \mathrm{d} \mathbf{s} .
$$

We can go on with this process and find explicitly functions $\mathbf{Z}_{i, \text { int }}^{2}(\mathbf{s})$ for $i=2, \ldots, 5$ such that

$$
\forall i \in\{2, \ldots, 5\}, \quad \mathbf{Z}_{i}^{2}=-\varepsilon \int_{\partial \mathbb{R}_{+}^{3}} h(\mathbf{s})\left(\mathbf{Z}_{i, i n t}^{2}(\mathbf{s})\right) \mathrm{d} \mathbf{s} .
$$

Finally,

$$
\mathcal{B}^{h}(\mathbf{X})=-\varepsilon^{2} \int_{\partial \mathbb{R}_{+}^{3}} \int_{\partial \mathbb{R}_{+}^{3}} h(\mathbf{s}) h\left(\mathbf{s}^{\prime}\right) \sum_{\mathcal{I}} \pm\left|\mathbf{Z}_{k_{1}, \text { int }}^{2}(\mathbf{s}) \mathbf{Z}_{k_{2}, \text { int }}^{2}\left(\mathbf{s}^{\prime}\right) \mathbf{Z}_{k_{3}}^{1} \mathbf{Z}_{k_{4}}^{1} \mathbf{Z}_{k_{5}}^{1}(\mathbf{X})\right| \mathrm{d} \mathbf{s} \mathrm{d} \mathbf{s}^{\prime} .
$$

We call detint the function defined by,

$$
\operatorname{det}_{\text {int }}:\left(\mathbf{X}, \mathbf{s}, \mathbf{s}^{\prime}\right) \mapsto \sum_{\mathcal{I}} \pm\left|\mathbf{Z}_{k_{1}, \text { int }}^{2}(\mathbf{s}) \mathbf{Z}_{k_{2}, \text { int }}^{2}\left(\mathbf{s}^{\prime}\right) \mathbf{Z}_{k_{3}}^{1} \mathbf{Z}_{k_{4}}^{1} \mathbf{Z}_{k_{5}}^{1}(\mathbf{X})\right|
$$

Clearly, for Theorem 4.10 to hold, it is enough that there exists $\mathbf{X}_{0}$ and $\left(\mathbf{s}, \mathbf{s}^{\prime}\right) \in\left(\partial \mathbb{R}_{+}^{3}\right)^{2}$ such that $\operatorname{det}_{\text {int }}\left(\mathbf{X}_{0}, \mathbf{s}, \mathbf{s}^{\prime}\right)$ is not zero for some $\left(\mathbf{s}, \mathbf{s}^{\prime}\right) \in \mathbb{R}^{4}$. Indeed, we can then adjust the function $h$ to make the integral non-zero. The calculation of detint can be carried out using Maple. More precisely, one can derive an equivalent as $z$ goes to infinity, and check that $\operatorname{det}_{\text {int }}\left(\mathbf{X}_{0}, \cdot, \cdot\right) \neq 0$ for $\mathbf{X}_{0}=\left(1,2, \frac{\pi}{3}, \frac{\pi}{3}, 1,2, z\right)$ for $z$ large enough (see appendix for details).

Finally, we define the surface $h$ as a cut off function $\chi_{\mathbf{x}_{0}} \in C_{c}^{\infty}\left(\mathbb{R}^{2}\right)$ such that $\chi_{\mathbf{x}_{0}}=1$ near $(x, y)=(1,2)$. It concludes the proof of Proposition 4.10.

As a direct consequence of Proposition 4.10 and the analyticity of the function det, for almost every $(\varepsilon, a, \mathbf{X}) \in$ $\mathbb{R}^{2} \times \mathcal{M}$, the Lie algebra of the vector fields $\mathbf{F}_{1}$ and $\mathbf{F}_{2}$ is fully generated. Finally, by using Chow's theorem (the theorem is stated in Appendix B), we get Theorem 2.4.

\section{Conclusions And Perspectives}

The aim of this present paper was to examine how the controllability of low Reynolds number artificial swimmers is affected by the presence of a rough wall on a fluid. This study generalizes the one made by Alouges and Giraldi in [2] which deals with the effect of a plane wall on the controllability of this particular swimmers.

Firstly, we show Theorem 2.1. It deals with the regularity of the dynamics of the swimmers. Indeed, we prove that the equation of motion of such particular swimmers are analytic with respect to the parameters defining the swimmer (radius of the ball, position and length of the arms) and the typical height of roughness of the wall. Then, we deduce Theorem 2.3 which claims that the 4-sphere swimmer remains controllable with the presence of roughness. The proof is based on general arguments which could be used for other models of micro-swimmer.

Secondly, Theorem 2.4 examines the controllability of the Three-sphere swimmer in the presence of a rough wall. More precisely, we show that there exists a roughness such that the swimmer can locally reach any direction. We recall that the previous studies made on the 3-sphere swimmer allow to show that it can reach only one direction (see [4]) when it evolves in a whole space and three directions with the presence of a plane wall (see [2]). In our case, the roughness leads to break the symmetry of the system "fluids-swimmer". As a result, it allows the swimmer to reach any direction. The proof is an in-depth study which associates several tools both in hydrodynamics and control theory. The general "idea" emphasizes here is the fact that in the real life all the micro-organism, regardless how symmetric it is, can move in any direction. 
The quantitative approach to this question together with the complete understanding in a view of controllability of underlying systems is far beyond reach and thus still under progress as in a another direction, the consideration of an confined environment, e.g. when the fluid is bounded. Future work will also explore which are the directions easier to reach than the others by varying the rough wall.

\section{Appendix A: Well-Posedness Result for the Stokes System}

We show here the well-posedness of the inhomogeneous Stokes system involved in the proof of Theorem 2.1. We refer to this proof for notations, and shall drop here all bars for brevity. What we want to show is

Theorem A.1. Let $\left(F, G, V_{1}, \ldots, V_{N}\right)$ given in $\mathcal{V}^{\prime} \times L^{2}(\mathcal{F}) \times \prod_{l} H^{1 / 2}\left(B_{l}\right)$. There exists a unique solution $(V, Q)$ in $\mathcal{V}_{0} \times L^{2}(\mathcal{F})$ of

$$
\begin{aligned}
&-\Delta V+\nabla Q=F \text { in } \mathcal{F}, \\
& \operatorname{div} V=G \quad \text { in } \mathcal{F}, \\
& V=V_{l} \quad \text { at } \partial B_{l}, \quad l=1 \ldots N .
\end{aligned}
$$

We recall that the space $\mathcal{V}_{0}$ encodes the additional homogeneous Dirichlet condition at $\partial \mathcal{O}$.

Proof of the Theorem. The theorem follows from

Proposition A.2. For all $\left(G, V_{1}, \ldots, V_{n}\right)$ given in $L^{2}(\mathcal{F}) \times \prod_{l} H^{1 / 2}\left(B_{l}\right)$, there exists a field $W \in \mathcal{V}_{0}$ satisfying

$$
\operatorname{div} W=G \quad \text { in } \mathcal{F}, \quad W=V_{l} \quad \text { at } \partial B_{l}, \quad l=1 \ldots N .
$$

together with the estimate: $\|\nabla W\|_{L^{2}} \leqslant C\left(\|G\|_{L^{2}}+\sum_{l=1}^{N}\left\|V_{l}\right\|_{H^{1 / 2}}\right)$.

This proposition will be proved below. Let us explain how it implies the theorem. First, considering $V^{\prime}:=$ $V-W$, and $F^{\prime}:=F+\Delta W$ one can come down to the homogeneous case $G=0$ and $V_{l}=0$ for all $l$. The homogeneous case can then be solved by a standard application of Lax-Milgram theorem. More precisely, defining

$$
\mathcal{V}_{\text {hom,div }}:=\left\{V^{\prime} \in \mathcal{V}_{0},\left.\quad V^{\prime}\right|_{\partial B_{l}}=0 \forall l, \quad \operatorname{div} V^{\prime}=0\right\}
$$

one can show easily that there is a unique $V^{\prime} \in \mathcal{V}_{\text {hom,div }}$ satisfying

$$
\int \nabla V^{\prime} \cdot \nabla \varphi=<F, \varphi>, \quad \forall \varphi \in \mathcal{V}_{\text {hom,div }}
$$

We recall that the condition $V /(1+|\mathbf{x}|) \in L^{2}(\mathcal{F})$ in the definition of $\mathcal{V}_{0}$ is related to the Hardy inequality.

By standard arguments, one then recovers a pressure field $Q \in L_{\text {loc }}^{2}(\mathcal{F})$ so that the Stokes equation $-\Delta V^{\prime}+$ $\nabla Q=F^{\prime}$ holds. Eventually, to show that we can take $Q$ in $L^{2}(\mathcal{F})$, we invoke ([9], Thm. 3.5.3, p. 217): it is enough that for all $G \in L^{2}(\mathcal{F})$, the problem

$$
\operatorname{div} W=G \text { in } \mathcal{F},\left.\quad W\right|_{\partial \mathcal{F}}=0
$$

has a solution $W \in \mathcal{V}_{0}$ with $\|\nabla W\|_{L^{2}} \leqslant C\|G\|_{L^{2}}$. This is a special case of Proposition A.2. This ends the proof.

Proof of the Proposition. Again, we single out the key ingredient in a

Lemma A.3. Given $G \in L^{2}(\mathcal{O})$, there exists a field $W \in \mathcal{V}_{0}$ such that

$$
\operatorname{div} W=G,\left.\quad W\right|_{\partial \mathcal{O}}=0 \quad\|\nabla W\|_{L^{2}} \leqslant C\|G\|_{L^{2}} .
$$


Note that this lemma is only about the domain $\mathcal{O}$, that is without the balls. Let us postpone its proof, and show how it implies the existence of a $W$ satisfying (A.1).

- First step: we lift the boundary data $V_{l}$. One can find $\bar{W} \in H^{1}(\mathcal{F})$ compactly supported near the balls, such that $\bar{W}=V_{l}$ at $\partial B_{l}$. Up to replace $W$ by $W-\bar{W}$ and $G$ by $G-\operatorname{div} \bar{W}$, we can assume $V_{l}=0$ for all $l$.

- Second step (assuming now $V_{l}=0$ for all $l$ ): we extend $G$ by 0 in the balls and apply the Lemma: it provides a $\tilde{W}$ satisfying $\operatorname{div} \tilde{W}=G,\left.\quad \tilde{W}\right|_{\partial \mathcal{O}}=0$. However, the boundary data at the balls is non-zero: $\left.\tilde{W}\right|_{\partial B_{l}} \neq 0$.

- Third step: we correct this non-zero boundary data. We observe that

$$
\int_{\partial B_{l}} \tilde{W} \cdot n \mathrm{~d} s=0=\int_{B_{l}} \operatorname{div} \tilde{W}=0
$$

as $G$ was extended by zero inside the balls. Thanks to this "compatibility" condition, we can use a standard result of Bogovskii (see [9], Exercice III.3.5, p. 176): for all $l$, there exists a field $W_{l}$ defined over the annulus $\left\{a<\left|x-x_{l}\right|<a+\eta\right\}$, satisfying

$$
\operatorname{div} W_{l}=0,\left.\quad W_{l}\right|_{\partial B_{l}}=-\left.\tilde{W}\right|_{\partial B_{l}},\left.\quad W_{l}\right|_{\left\{\left|x-x_{l}\right|=a+\eta\right\}}=0 .
$$

We take $\eta$ small enough so that the annuli do not intersect. Then, we extend the $W_{l}$ 's by 0 outside the annuli and set $W:=\tilde{W}+\sum W_{l}$. This new field $W$ satisfies (A.1), as expected.

Proof of the Lemma. In the case where $h=$ cst., that is for a flat half-space, the result is classical (cf. [9], Cor. 4.3.1, p. 261). In particular, if the support of $G$ is included in $\left\{x_{3}>\sup |h|\right\}$, the problem is solved: one can take the solution $W$ of

$$
\operatorname{div} W=G \quad \text { for } \quad x_{3}>\sup |h|,\left.\quad W\right|_{\left\{x_{3}=\sup |h|\right\}}=0
$$

and extend it by zero below $\left\{x_{3}=\sup |h|\right\}$.

For general $G$, we can decompose $\left.G=G 1_{\left\{x_{3}>\sup |h|\right.}\right\}+G 1_{\left\{x_{3}<\sup |h|\right\}}$, and handle the first part as previously.

In other words, it remains to consider the case where $G$ is compactly supported in $x_{3}$. From there, we proceed in three steps:

- Step 1. Let $R$ such that $G=0$ for $x_{3} \geqslant R$. We introduce $W^{1}:=\nabla \psi 1_{\left\{x_{3}<R\right\}}$ where $\psi$ satisfies

$$
\Delta \psi=G \quad \text { for } \varepsilon h<x_{3}<R,\left.\quad \partial_{n} \psi\right|_{\partial \mathcal{O}}=0,\left.\quad \psi\right|_{x_{3}=R}=0 .
$$

This Poisson equation has a unique solution in $H^{2}\left(\left\{\varepsilon h<x_{3}<R\right\}\right)$ : note that Poincaré inequality applies thanks to the Dirichlet condition at $x_{3}=R$. Hence, $W^{1}$ satisfies $\operatorname{div} W^{1}=G$ in the strip $\left\{\varepsilon h<x_{3}<R\right\}$, and also trivially in the half-space $\left\{x_{3}>R\right\}$. However, two problems remain: the normal component of $W^{1}$ jumps at $x_{3}=R$, and it has non-zero boundary data at $\left\{x_{3}=\varepsilon h\right\}$.

- Step 2. Correction of the jump at $x_{3}=R$. We just introduce the field $W^{2}:=\tilde{W} 1_{\left\{x_{3}>R\right\}}$, where $\tilde{W}$ satisfies

$$
\operatorname{div} \tilde{W}=0 \quad \text { for } \quad x_{3}>R,\left.\quad \tilde{W}\right|_{\left\{x_{3}=R\right\}}=\left.\nabla \Psi\right|_{x_{3}=R},
$$

$$
\|\nabla \tilde{W}\|_{L^{2}} \leqslant C\|\nabla \psi\|_{H^{1 / 2}\left(\left\{x_{3}=R\right\}\right)}\left(\leqslant C\|G\|_{L^{2}}\right) .
$$

The existence of such $\tilde{W}$ is classical (see [9], Thm. IV.3.3).

- Step 3. Correction of the boundary data. Thanks to the Neumann condition on $\psi$, we have $\left.W^{1} \cdot n\right|_{\partial \mathcal{O}}=0$. We introduce some partition of unity $\left(\chi_{k}=\chi_{k}\left(x_{1}, x_{2}\right)\right)_{k \in \mathbf{Z}^{2}}$ associated to a covering of $\mathbb{R}^{2}$ by rectangles $R_{k}$. More precisely, we assume that the lengths of $R_{k}$ are uniformly bounded in $k$, and that the $C^{1}$ norms of $\chi^{k}$ are uniformly bounded in $k$ (we leave the construction of examples to the reader). Thanks to the 
tangency condition on $W^{1}$, we can apply the Bogovskii's result seen above on slices $S_{k}:=\left\{\left(x_{1}, x_{2}\right) \in\right.$ $\left.R_{k}, \quad \varepsilon h\left(x_{1}, x_{2}\right)<x_{3}<R\right\}, k \in \mathbf{Z}^{2}$. Hence, there exists some $W_{k} \in H^{1}\left(S_{k}\right)$ such that

$$
\operatorname{div} W_{k}=0 \quad \text { in } \quad S_{k}, \quad W_{k}=-\chi_{k} W^{1} \quad \text { at } \quad \partial S_{k} \cap \partial \mathcal{O}, \quad W_{k}=0 \quad \text { at } \quad \partial S_{k} \backslash \mathcal{O},
$$

and $\left\|\nabla W_{k}\right\|_{L^{2}} \leqslant C\left\|\chi_{k} W^{1}\right\|_{H^{1 / 2}(\partial \mathcal{O})}$. Extending all $W_{k}$ 's by 0 outside $S_{k}$, and setting $W^{3}:=\sum_{k \in \mathbf{Z}^{2}} W_{k}$, we find that

$$
\operatorname{div} W^{3}=0 \quad \text { in } \quad \mathcal{O},\left.\quad W^{3}\right|_{\partial \mathcal{O}}=-\left.W^{1}\right|_{\partial \mathcal{O}}, \quad\left\|\nabla W^{3}\right\|_{L^{2}(\mathcal{O})} \leqslant C\left\|W^{1}\right\|_{H^{1 / 2}(\partial \mathcal{O})}\left(\leqslant C\left\|W^{1}\right\|_{H^{1}(\mathcal{O})}\right) .
$$

Finally, $W=W^{1}+W^{2}+W^{3}$ fulfills all requirements, which concludes the proof of the lemma.

\section{Appendix B: On Chow's TheOREM}

Let us recall here some elements to understand Chow's theorem. More details can be found in the book of Jurdjevic (see [14]). Let $F$ and $G$ be two vector fields defined on a smooth finite dimensional manifold $\mathcal{M}$. The Lie bracket of $F$ and $G$ is the vector field defined at any point $X \in \mathcal{M}$ by $[F, G](X):=(F \cdot \nabla) G(X)-(G \cdot \nabla) F(X)$. For a family of vector fields $\mathcal{F}$ on $\mathcal{M}, \operatorname{Lie}(\mathcal{F})$ denotes the Lie algebra generated by $\mathcal{F}$. Namely, this is the smallest algebra - defined by the Lie bracket operation - which contains $\mathcal{F}$ (therefore $\mathcal{F} \subset$ Lie $(\mathcal{F})$ and for any two vectorfields $F \in \operatorname{Lie}(\mathcal{F})$ and $G \in \operatorname{Lie}(\mathcal{F})$, the Lie bracket $[F, G] \in \operatorname{Lie}(\mathcal{F})$ ). Eventually, for any point $X \in \mathcal{M}, \operatorname{Lie}_{X}(\mathcal{F})$ denotes the set of all tangent vectors $V(X)$ with $V$ in $\operatorname{Lie}(\mathcal{F})$. It follows that $\operatorname{Lie}_{X}(\mathcal{F})$ is a linear subspace of $T_{X} \mathcal{M}$ and is hence finite-dimensional.

Lie brackets and Lie algebras play a prominent role in finite dimensional control theory. An important example is Chow's theorem, which gives a small time local controllability result (this theorem is used throughout the whole paper):

Theorem B.1 ([8], p. 135). Let $\Omega$ be an nonempty open subset of $\mathbf{R}^{n}$, and let $\mathcal{F}=\left(F_{i}\right)_{i=1}^{m}$, a family of vector fields, such that $F_{i} \in C^{\infty}\left(\Omega, \mathbf{R}^{n}\right), \forall i \in\{1, \cdots, m\}$.

Let $X_{e}$ such that

$$
\operatorname{Lie}_{X_{e}}(\mathcal{F})=\mathbf{R}^{n} \text {. }
$$

Then, for every $\epsilon>0$, there exists a real number $\eta>0$ such that, for every $\left(X^{0}, X^{1}\right) \in\left\{X s . t .\left\|X-X_{e}\right\|<\eta\right\}^{2}$, there exists a bounded measurable function $u:[0, \epsilon] \rightarrow \mathbf{R}^{n}$ such that the solution of the Cauchy problem

$$
\left\{\begin{array}{l}
\dot{X}=\sum_{i=1}^{m} u_{i} F_{i}(X), \\
X(0)=X^{0}
\end{array}\right.
$$

is defined on $[0, \epsilon]$ and satisfies $X(\epsilon)=X^{1}$.

\section{Appendix C: Formal EXPRESSIONS}

We express here the requisite formal expression of the vector fields for the calculus of the detint at the point $\mathbf{X}_{0}=\left(1,2, \frac{\pi}{3}, \frac{\pi}{3}, 1,2, z\right)$.

First at all, we have used the software MAPLE to symbolically compute The vector fields $\mathbf{F}_{1}$ and $\mathbf{F}_{2}$ by using the formula (4.44). Then, we deduce the expression of every vector which belongs to the set $\mathcal{F}_{\text {cal }}:=$ $\left\{\mathbf{Z}_{k}^{j} \quad\right.$ s.t. $\left.\quad k=1, \cdots, 5 j=1,2\right\}$ defined in (4.46). In the following, we express the first asymptotic terms when $z$ goes to infinity of the vector fields which belong to $\mathcal{F}_{c a l}$ at $\mathbf{X}_{0}$. The asymptotic expression of the determinant detint, defined in (4.50), is deduced. 
- The expansion of vector fields $\mathbf{Z}_{1}^{1}$ and $\mathbf{Z}_{1}^{2}$ are expressed by,

$$
\begin{aligned}
& \mathbf{Z}_{1}^{1}\left(\mathbf{X}_{0}\right)=\left(\begin{array}{c}
\frac{21627}{57344} \frac{\sqrt{3}}{z^{4}}-\frac{237897}{802816} \frac{\sqrt{3}}{z^{5}}-\frac{56965095}{25690112} \frac{\sqrt{3}}{z^{6}}-\frac{29418201}{25690112} \frac{\sqrt{3}}{z^{7}}+\frac{141}{25088} \frac{\sqrt{3}}{z^{8}} \\
0 \\
\frac{41}{432} \sqrt{3}-\frac{7209}{229376} \frac{\sqrt{(3)}}{z^{4}}-\frac{923829}{3211264} \frac{\sqrt{(3)}}{z^{5}}+\frac{45738445}{102760448} \frac{\sqrt{3}}{z^{6}}-\frac{83758845}{102760448} \frac{\sqrt{(3)}}{z^{7}}-\frac{47}{100352} \frac{\sqrt{3}}{z^{8}} \\
\frac{41}{144}-\frac{2167}{229376 z^{4}}-\frac{2771487}{3211264 z^{5}}+\frac{137215335}{102760448 z^{6}}-\frac{251276535}{102760448 z^{7}}-\frac{141}{100352 z^{8}} \\
\frac{41}{216}+\frac{21627}{114688 z^{4}}-\frac{927033}{1605632 z^{5}}-\frac{38588135}{51380224 z^{6}}+\frac{191091795}{51380224 z^{7}}-\frac{61}{100352 z^{8}}
\end{array}\right), \\
& \mathbf{Z}_{1}^{2}\left(\mathbf{X}_{0}, s, s^{\prime}\right)=\left(\begin{array}{c}
\frac{2187}{448} \frac{\left(\frac{1}{2}+\frac{1}{2} \sqrt{3}\right)\left(1-\frac{1}{4} \sqrt{3}\right)}{\pi z^{7}} \\
-\frac{2187}{448} \frac{-\frac{1}{2} \sqrt{(3)+\frac{1}{2}}}{\pi z^{7}} \\
\frac{81}{448} \frac{\left(-\frac{39}{32} \sqrt{\left.(3)+\frac{81}{32}\right) \sqrt{(3)}}\right.}{\pi z^{7}} \\
-\frac{243}{448} \frac{-\frac{9}{32} \sqrt{3}+\frac{63}{32}}{\pi z^{7}} \\
\frac{729}{896} \frac{\left(\frac{1}{2}+\frac{1}{2} \sqrt{3}\right)\left(1-\frac{1}{4} \sqrt{3}\right) \sqrt{3}}{\pi z^{7}}
\end{array}\right) \\
& +\left(\begin{array}{c}
-\frac{27}{784} \frac{\sqrt{3}\left(\frac{19593}{64} v+\frac{6531}{64} s-\frac{567}{64} \sqrt{3} s^{\prime}-\frac{189}{64} \sqrt{3} s-\frac{927}{128} \sqrt{3}-\frac{63609}{64}\right)}{\pi z^{8}} \\
\frac{27}{784} \frac{\sqrt{3}\left(-\frac{6531}{16} s^{\prime}+\frac{7665}{16} s-\frac{189}{16} \sqrt{3} s^{\prime}-\frac{189}{16} \sqrt{3} s-\frac{2997}{32} \sqrt{3}+\frac{5727}{8}\right)}{\pi z^{8}} \\
-\frac{9}{196} \frac{\frac{8505}{1024} s^{\prime}+\frac{1587915}{1024} s+\frac{58779}{1024} \sqrt{3} s^{\prime}-\frac{3087}{4} s^{2}+\frac{230769}{1224} \sqrt{3} s-\frac{406647}{1024} \sqrt{3}-\frac{2126079}{2048}}{\pi z^{8}} \\
\frac{9}{196} \frac{-\frac{4011777}{1024} s^{\prime}-\frac{72387}{1024} s+\frac{567}{1024} \sqrt{3} s^{\prime}+\frac{3087}{4} s^{\prime 2}+\frac{1701}{1024} \sqrt{(3)} s+\frac{54351}{2048} \sqrt{3}+\frac{5792805}{1024}}{\pi z^{8}} \\
-\frac{27}{1568} \frac{\frac{19593}{64} s^{\prime}+\frac{6531}{64} s-\frac{567}{64} \sqrt{3} s^{\prime}-\frac{189}{64} \sqrt{3} s-\frac{927}{128} \sqrt{3}-\frac{63609}{64}}{\pi z^{8}}
\end{array}\right) \\
& +O\left(\frac{1}{z^{9}}\right) \text {. }
\end{aligned}
$$

- The expansion of vector fields $\mathbf{Z}_{2}^{1}$ and $\mathbf{Z}_{2}^{2}$ are expressed by,

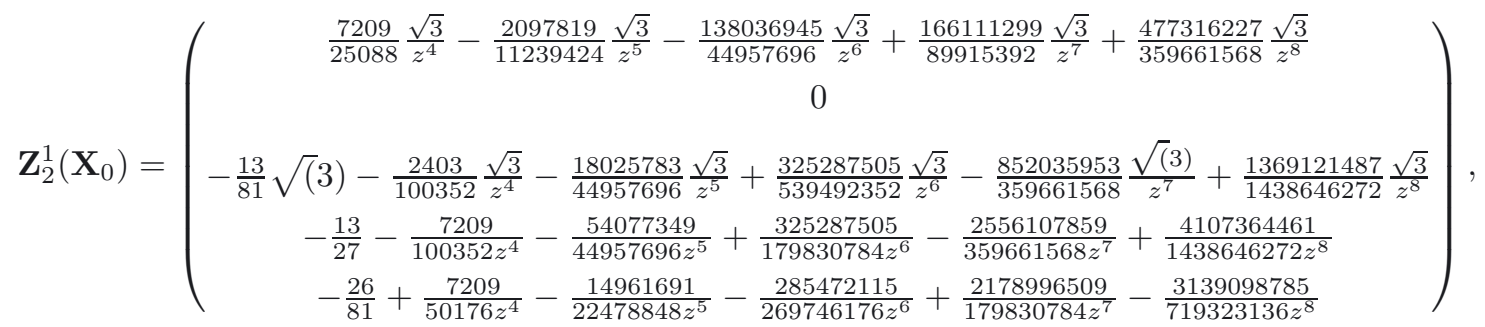

$$
\begin{aligned}
& \mathbf{Z}_{2}^{2}\left(\mathbf{X}_{0}, s, s^{\prime}\right)=\left(\begin{array}{c}
\frac{729}{196} \frac{\left(\frac{1}{2}+\frac{1}{2} \sqrt{3}\right)\left(1-\frac{1}{4} \sqrt{3}\right)}{\pi z^{7}} \\
-\frac{729}{196} \frac{-\frac{1}{2} \sqrt{3}+\frac{1}{2}}{\pi z^{7}} \\
\frac{9}{3136} \sqrt{3} \frac{\left(\frac{243}{2}-\frac{117}{2} \sqrt{3}\right)}{\pi z^{7}} \\
-\frac{27}{3136} \frac{-\frac{27}{2} \sqrt{3}+\frac{189}{2}}{\pi z^{7}} \\
\frac{243}{392} \frac{\left(\frac{1}{2}+\frac{1}{2} \sqrt{3}\right)\left(1-\frac{1}{4} \sqrt{3}\right) \sqrt{3}}{\pi z^{7}}
\end{array}\right)
\end{aligned}
$$




$$
\begin{gathered}
+\left(\begin{array}{c}
-\frac{9}{5488} \frac{\sqrt{3}\left(\frac{11403}{8} s^{\prime}+\frac{3801}{8} s-\frac{567}{4} \sqrt{3} s^{\prime}-\frac{189}{4} \sqrt{3} s+\frac{99591}{128} \sqrt{3}+\frac{26481}{64}\right)}{\pi z^{8}} \\
+\frac{9}{5488} \sqrt{3} \frac{\left(-4161-\frac{3801}{2} s^{\prime}+\frac{6069}{2} s-189 \sqrt{3} s^{\prime}-189 \sqrt{3} s+\frac{69765}{32} \sqrt{3}\right)}{\pi z^{8}} \\
-\frac{3}{1372} \frac{\frac{8505}{64} s^{\prime}+\frac{698859}{64} s+\frac{34209}{128} \sqrt{3} s^{\prime}-\frac{21609}{4} s^{2}-\frac{307125}{128} \sqrt{3} s+\frac{2517615}{1024} \sqrt{3}-\frac{23258601}{2048}}{\pi z^{8}} \\
+\frac{3}{1372} \frac{\left(-\frac{1994643}{128} s^{\prime}-\frac{61425}{128} s+\frac{567}{64} \sqrt{3} s^{\prime}+\frac{21609}{4} s^{\prime 2}+\frac{1701}{64} \sqrt{3} s+\frac{54297}{2048} \sqrt{3}+\frac{26018883}{1024}\right)}{\pi z^{8}} \\
+\frac{9}{10976} \frac{\left(-\frac{11403}{8} s^{\prime}-\frac{3801}{8} s+\frac{567}{4} \sqrt{3} s^{\prime}+\frac{189}{4} \sqrt{3} s-\frac{26481}{64}-\frac{99591}{128} \sqrt{3}\right)}{\pi z^{8}}
\end{array}\right) \\
+\quad O\left(\frac{1}{z^{9}}\right) .
\end{gathered}
$$

- The expansion of vector fields $\mathbf{Z}_{3}^{1}$ and $\mathbf{Z}_{3}^{2}$ are expressed by,

$$
\begin{aligned}
& \mathbf{Z}_{3}^{1}\left(\mathbf{X}_{0}\right)=\left(\begin{array}{c}
\frac{36045}{802816} \frac{\sqrt{3}}{z^{4}}-\frac{1737369}{22478848} \frac{s q r t 3}{z^{5}}-\frac{688526655}{359661568} \frac{\sqrt{3}}{z^{6}}-\frac{1554687891}{359661568} \frac{\sqrt{3}}{z^{7}}-\frac{473853315}{359661568} \frac{\sqrt{3}}{z^{8}} \\
0 \\
-\frac{19}{1296} \sqrt{3}-\frac{12015}{3211264} \frac{\sqrt{3}}{z^{4}}-\frac{22827197}{89915392} \frac{\sqrt{3}}{z^{5}}+\frac{16247794555}{4315938816} \frac{\sqrt{3}}{z^{6}}-\frac{975961023}{1438646272} \frac{\sqrt{(3)}}{z^{7}}-\frac{1370275791}{1438646272} \frac{\sqrt{3}}{z^{8}} \\
-\frac{19}{432}-\frac{36045}{3211264 z^{4}}-\frac{68481591}{89915392 z^{5}}+\frac{1624779455}{1438646272 z^{6}}-\frac{2927883069}{1438646272 z^{7}}-\frac{4110827373}{1438646272 z^{8}} \\
-\frac{19}{648}+\frac{36045}{1605632 z^{4}}-\frac{17817209}{44957696 z^{5}}-\frac{1422366365}{2157969408 z^{6}}+\frac{2266030269}{719323136 z^{7}}+\frac{3127809953}{719323136 z^{8}}
\end{array}\right), \\
& \mathbf{Z}_{3}^{2}\left(\mathbf{X}_{0}, s, s^{\prime}\right)=\left(\begin{array}{c}
\frac{3645}{6272} \frac{\left(\frac{1}{2}+\frac{1}{2} \sqrt{3}\right)\left(1-\frac{1}{4} \sqrt{3}\right)}{\pi z^{7}} \\
-\frac{3645}{6272} \frac{\left(-\frac{1}{2} \sqrt{3}+\frac{1}{2}\right)}{\pi z^{7}} \\
\frac{9}{3136} \sqrt{3} \frac{\left(-\frac{585}{64} \sqrt{3}+\frac{1215}{64}\right)}{\pi z^{7}} \\
-\frac{27}{3136} \frac{\left(\frac{945}{64}-\frac{135}{64} \sqrt{3}\right)}{\pi z^{7}} \\
\frac{1215}{12544} \frac{\left(\frac{1}{2}+\frac{1}{2} \sqrt{3}\right)\left(1-\frac{1}{4} \sqrt{3}\right) \sqrt{3}}{\pi z^{7}}
\end{array}\right) \\
& +\left(\begin{array}{c}
-\frac{9}{5488} \sqrt{3} \frac{\left(\frac{320229}{128} s^{\prime}+\frac{106743}{128} s-\frac{2835}{128} \sqrt{3} s^{\prime}-\frac{945}{128} \sqrt{3} s-\frac{48927}{64} \sqrt{3}-\frac{214935}{16}\right)}{\pi z^{8}} \\
-\frac{9}{5488} \sqrt{3} \frac{\left(\frac{106743}{32} s^{\prime}-\frac{112413}{32} s+\frac{945}{32} \sqrt{3} s^{\prime}-\frac{213405}{16}+\frac{945}{32} \sqrt{3} s+\frac{53283}{16} \sqrt{3}\right)}{\pi z^{8}} \\
-\frac{3}{1372} \frac{\left(\frac{42525}{2048} s^{\prime}+\frac{22164471}{2048} s+\frac{960687}{2048} \sqrt{3} s^{\prime}-\frac{21609}{4} s^{2}+\frac{11452077}{2048} \sqrt{3} s-\frac{2054187}{256} \sqrt{3}-\frac{10591731}{1024}\right)}{\pi z^{8}} \\
-\frac{3}{1372} \frac{\left(\frac{80736957}{2048} s^{\prime}+\frac{1028727}{2048} s-\frac{2835}{2048} \sqrt{3} s^{\prime}-\frac{21609}{4} s^{\prime 2}-\frac{8505}{2048} \sqrt{3} s-\frac{19271313}{256}-\frac{677835}{1024} \sqrt{3}\right)}{\pi z^{8}} \\
-\frac{9}{10976} \frac{\left(\frac{320229}{128} s^{\prime}+\frac{106743}{128} s-\frac{2885}{128} \sqrt{3} s^{\prime}-\frac{945}{128} \sqrt{3} s-\frac{48927}{64} \sqrt{3}-\frac{214935}{16}\right)}{\pi z^{8}}
\end{array}\right) \\
& +O\left(\frac{1}{z^{9}}\right) \text {. }
\end{aligned}
$$


- The expansion of vector fields $\mathbf{Z}_{4}^{1}$ and $\mathbf{Z}_{4}^{2}$ are expressed by,

$$
\begin{aligned}
& \mathbf{Z}_{4}^{1}\left(\mathbf{X}_{0}\right)=\left(\begin{array}{c}
-\frac{64881}{351232} \frac{\sqrt{3}}{z^{4}}+\frac{69687}{307328} \frac{\sqrt{3}}{z^{5}}-\frac{1076724525}{629407744} \frac{\sqrt{3}}{z^{6}}+\frac{5383125903}{629407744} \frac{\sqrt{3}}{z^{7}}-\frac{4471078809}{2517630976} \frac{\sqrt{3}}{z^{8}} \\
0 \\
\frac{40}{81} \sqrt{3}+\frac{21627}{1404928} \frac{\sqrt{3}}{z^{4}}-\frac{2233885}{9834496} \frac{\sqrt{3}}{z^{5}}+\frac{23874784445}{7552892928} \frac{\sqrt{3}}{z^{6}}-\frac{28725839983}{7552892928} \frac{\sqrt{3}}{z^{7}}+\frac{50480747763}{10070523904} \frac{\sqrt{3}}{z^{8}} \\
\frac{40}{27}+\frac{64881}{1404928 z^{4}}-\frac{6701655}{9834496 z^{5}}+\frac{2387478445}{2517630976 z^{6}}-\frac{28725839983}{2517630976 z^{7}}+\frac{151442243289}{10070523904 z^{8}} \\
\frac{80}{81}-\frac{64881}{702464 z^{4}}-\frac{424745}{2458624 z^{5}}-\frac{2329702535}{3776446464 z^{6}}+\frac{714414731499}{3776446464 z^{7}}-\frac{13859698939}{5035261952 z^{8}}
\end{array}\right), \\
& \mathbf{Z}_{4}^{2}\left(\mathbf{X}_{0}, s, s^{\prime}\right)=\left(\begin{array}{c}
-\frac{6561}{2744} \frac{\left(\frac{1}{2}+\frac{1}{2} \sqrt{3}\right)\left(1-\frac{1}{4} \sqrt{3}\right)}{\pi z^{7}} \\
\frac{6561}{2744} \frac{\left(-\frac{1}{2} \sqrt{3}+\frac{1}{2}\right)}{\pi z^{7}} \\
\frac{6561}{2744} \frac{\left(-\frac{1}{2} \sqrt{3}+\frac{1}{2}\right)}{\pi z^{7}} \\
-\frac{27}{21952} \frac{\left(\frac{729}{4} \sqrt{3}-\frac{1053}{4}\right)}{\pi z^{7}} \\
-\frac{27}{21952} \sqrt{3} \frac{\left(-\frac{567}{4} \sqrt{3}+\frac{243}{4}\right)}{\pi z^{7}}
\end{array}\right) \\
& +\left(\begin{array}{c}
-\frac{27}{38416}\left(\frac{1}{2}+\frac{1}{2} \sqrt{3}\right)\left(1-\frac{1}{4} \sqrt{3}\right) \frac{\left(5103 s^{\prime}+1701 s+74197 / 4-\frac{1839}{4} \sqrt{3}\right)}{\pi z^{8}} \\
+\frac{27}{38416} \frac{\left(\frac{1701}{2} s^{\prime}+\frac{1701}{2} s-\frac{5103}{2} \sqrt{3} s^{\prime}+\frac{1701}{2} \sqrt{3} s-\frac{22411}{2} \sqrt{3}+\frac{53465}{4}\right)}{\pi z^{8}} \\
+\frac{27}{38416} \frac{\left(\frac{1701}{2} s^{\prime}+\frac{1701}{2} s-\frac{5103}{2} \sqrt{3} s^{\prime}+\frac{1701}{2} \sqrt{3} s-\frac{22411}{2} \sqrt{3}+\frac{53465}{4}\right)}{\pi z^{8}} \\
+\frac{1}{19208} \sqrt{3} \frac{\left(-\frac{413343}{64} s^{\prime}+\frac{7490259}{64} s+\frac{76545}{64} \sqrt{3} s^{\prime}+\frac{66339}{64} \sqrt{3} s+\frac{7226181}{128} \sqrt{3}-\frac{4527333}{32}\right)}{\pi z^{8}} \\
-\frac{3}{19208} \frac{\left(-\frac{7582113}{64} s^{\prime}+\frac{15309}{64} s+/ \text { frac } 510364 \sqrt{3} s^{\prime}+\frac{15309}{64} \sqrt{3} s+\frac{295803}{128} \sqrt{3}+\frac{2697651}{32}\right)}{\pi z^{8}}
\end{array}\right) \\
& +O\left(\frac{1}{z^{9}}\right) \text {. }
\end{aligned}
$$

- The expansion of vector fields $\mathbf{Z}_{5}^{1}$ and $\mathbf{Z}_{5}^{2}$ are expressed by,

$$
\mathbf{Z}_{5}^{1}\left(\mathbf{X}_{0}\right)=\left(\begin{array}{c}
-\frac{64881}{1404928} \frac{\sqrt{3}}{z^{4}}+\frac{5000643}{157351936} \frac{\sqrt{3}}{z^{5}}-\frac{1007277825}{1258815488} \frac{\sqrt{3}}{z^{6}}-\frac{7453380147}{1258815488} \frac{\sqrt{3}}{z^{7}}-\frac{9398907099}{1258815488} \frac{\sqrt{3}}{z^{8}} \\
0 \\
\frac{65}{2592} \sqrt{3}+\frac{21627}{5619712} \frac{\sqrt{3}}{z^{4}}-\frac{67204577}{629407744} \frac{\sqrt{3}}{z^{5}}+\frac{2318031745}{15105785856} \frac{\sqrt{3}}{z^{6}}-\frac{1808333293}{15105785856} \frac{\sqrt{3}}{z^{7}}-\frac{31796394461}{15105785856} \frac{\sqrt{3}}{z^{8}} \\
\frac{65}{864}+\frac{64881}{5619712 z^{4}}-\frac{201613731}{629407744 z^{5}}+\frac{2318031745}{5035261952 z^{6}}-\frac{1803333293}{5035261952 z^{7}}-\frac{31796394461}{5035261952 z^{8}} \\
\frac{65}{1296}-\frac{64881}{2809856 z^{4}}-\frac{40022909}{314703872 z^{5}}-\frac{2121362435}{7552892928 z^{6}}+\frac{1191556679}{7552892928 z^{7}}+\frac{62259224983}{7552892928 z^{8}}
\end{array}\right),
$$




$$
\begin{aligned}
& \mathbf{Z}_{5}^{2}\left(\mathbf{X}_{0}, s, s^{\prime}\right)=\left(\begin{array}{c}
-\frac{6561}{10976} \frac{\left(\frac{1}{2}+\frac{1}{2} \sqrt{3}\right)\left(1-\frac{1}{4} \sqrt{3}\right)}{\pi z^{7}} \\
\frac{6561}{10976} \frac{\left(-\frac{1}{2} \sqrt{3}+\frac{1}{2}\right)}{\pi z^{7}} \\
-\frac{27}{21952} \frac{\left(\frac{729}{16} \sqrt{3}-\frac{1053}{16}\right)}{\pi z^{7}} \\
\frac{27}{21952} \sqrt{3} \frac{\left(\frac{567}{16} \sqrt{3}-\frac{243}{16}\right)}{\pi z^{7}} \\
-\frac{2187}{21952} \frac{\left(\frac{1}{2}+\frac{1}{2} \sqrt{3}\right)\left(1-\frac{1}{4} \sqrt{3}\right) \sqrt{3}}{\pi z^{7}}
\end{array}\right) \\
& +\left(\begin{array}{c}
+\frac{27}{38416} \frac{\left(\frac{1}{2}+\frac{1}{2} \sqrt{3}\right)\left(1-\frac{1}{4} \sqrt{3}\right)\left(-\frac{5103}{4} s^{\prime}-\frac{1701}{4} s+\frac{2463}{64} \sqrt{3}+\frac{1431799}{64}\right)}{\pi z^{8}} \\
-\frac{27}{38416} \frac{\left(-\frac{1701}{8} s^{\prime}-\frac{1701}{8} s+\frac{5103}{8} \sqrt{3} s^{\prime}-\frac{1701}{8} \sqrt{3} s-\frac{171863}{16} \sqrt{3}+\frac{657773}{64}\right)}{\pi z^{8}} \\
+\frac{1}{19208} \sqrt{3} \frac{\left(-\frac{413343}{256} s^{\prime}-\frac{28812861}{256} s+\frac{76545}{256} \sqrt{3} s^{\prime}+\frac{66339}{26} \sqrt{3} s+\frac{102986487}{2048} \sqrt{3}+\frac{144659439}{1024}\right)}{\pi z^{8}} \\
-\frac{3}{19208} \frac{\left(\frac{28721007}{256} s^{\prime}+\frac{15309}{256} s+\frac{5103}{256} \sqrt{3} s^{\prime}+\frac{15309}{256} \sqrt{3} s-\frac{7786791}{2048} \sqrt{3}-\frac{403566543}{1024}\right)}{\pi z^{8}} \\
+\frac{9}{76832} \frac{\left(\frac{1}{2}+\frac{1}{2} \sqrt{3}\right)\left(1-\frac{1}{4} \sqrt{3}\right) \sqrt{3}\left(-\frac{5103}{4} s^{\prime}-\frac{1701}{4} s+\frac{2463}{64} \sqrt{3}+\frac{1431799}{64}\right)}{\pi z^{8}}
\end{array}\right) \\
& +O\left(\frac{1}{z^{9}}\right) \text {. }
\end{aligned}
$$

Then, we express the function detint computed at $\left(\mathbf{X}_{0}, s, s^{\prime}, t, t^{\prime}\right)$

$$
\begin{aligned}
\operatorname{det}\left(\mathbf{X}_{0}, s, s^{\prime}, t, t^{\prime}\right)= & -\frac{4209544161}{5279854836580352}\left(-623289 s^{\prime}+623289 t+3220141 t^{\prime}\right. \\
& -3220141 s+1153029 s^{2}+384343 s^{\prime 2} \sqrt{3} \\
& +623289 \sqrt{3} s^{\prime}-384343 s^{2}-384343 \sqrt{3} s^{\prime 2} \\
& -623289 \sqrt{3} t-1682769 \sqrt{3} t^{\prime}+1682769 \sqrt{3} s \\
& \left.+384343 t^{\prime 2} \sqrt{3}+384343 \sqrt{3} t^{2}-1153029 t^{\prime 2}-384343 t^{2}\right) \frac{1}{\pi^{2} z^{24}} \\
& +O\left(\frac{1}{z^{25}}\right) .
\end{aligned}
$$

This formal expressions lead to conclude the proof of Theorem 2.4.

\section{REFERENCES}

[1] F. Alouges, A. DeSimone and A. Lefebvre, Optimal strokes for low Reynolds number swimmers : an example. J. Nonlinear Sci. 18 (2008) 277-302.

[2] F. Alouges and L. Giraldi, Enhanced controllability of low Reynolds number swimmers in the presence of a wall. Acta Applicandae Mathematicae 128 (2013) 153-179.

[3] F. Alouges, A. DeSimone, L. Giraldi and M. Zoppello, Self-propulsion of slender micro-swimmers by curvature control: N-link swimmers. J. Non-Linear Mech. 56 (2013) 132-141.

[4] F. Alouges, A. DeSimone, L. Heltai, A. Lefebvre and B. Merlet, Optimally swimming Stokesian robots. Discrete Contin. Dyn. Syst. Ser. B 18 (2013).

[5] A.P. Berke, L. Turner, H.C. Berg and E. Lauga, Hydrodynamic attraction of swimming microorganisms by surfaces. Phys. Rev. Lett. 101 (2008).

[6] J.R. Blake, A note on the image system for a Stokeslet in a no-slip boundary. Math. Proc. Cambridge Philos. Soc. 70 (1971) 303.

[7] T. Chambrion and A. Munnier, Locomotion and control of a self-propelled shape-changing body in a fluid. J. Nonlinear Sci. 21 (2011) 325-385. 
[8] J.M. Coron, Control and Nonlinearity. American Mathematical Society (2007).

[9] G.P. Galdi, An Introduction to the Mathematical Theory of the Navier-Stockes Equations. Springer (2011).

[10] D. Gérard Varet and M. Hillairet, Computation of the drag force on a rough sphere close to a wall. ESAIM: M2AN 46 (2012) 1201-1224.

[11] L. Giraldi, P. Martinon and M. Zoppello, Controllability and optimal strokes for N-link micro-swimmer. Proc. of 52th Conf. on Decision and Control (Florence, Italy) (2013).

[12] R. Golestanian and A. Ajdari, Analytic results for the three-sphere swimmer at low Reynolds. Phys. Rev. E 77 (2008) 036308.

[13] A. Henrot and M. Pierre, Variation et optimisation de formes. Une analyse géométrique. [A geometric analysis]. Vol. 48 of Math. Appl. Springer, Berlin, 2001.

[14] V. Jurdjevic, Geometric control theory. Cambridge University Press (1997).

[15] E. Lauga and T. Powers, The hydrodynamics of swimming micro-organisms. Rep. Prog. Phys. 72 (2009) 09660.

[16] R. Ledesma-Aguilar and J.M. Yeomans, Enhanced motility of a microswimmer in rigid and elastic confinement. Phys. Rev. Lett. 111 (2013) 138101

[17] J. Lighthill, Mathematical biofluiddynamic. Soc. Ind. Appl. Math. Philadelphia, Pa. (1975)

[18] J. Lohéac and A. Munnier, Controllability of 3D low Reynolds swimmers. ESAIM: COCV 20 (2014) $236-268$.

[19] J. Lohéac, J.F. Scheid and M. Tucsnak, Controllability and time optimal control for low Reynolds numbers swimmers. Acta Appl. Math. 123 (2013) 175-200.

[20] R. Montgomery, A tour of subriemannian geometries, theirs geodesics and applications. American Mathematical Society (2002).

[21] A. Najafi and R. Golestanian, Simple swimmer at low Reynolds number: Three linked spheres. Phys. Rev. E 69 (2004) 062901.

[22] Y. Or and M. Murray, Dynamics and stability of a class of low Reynolds number swimmers near a wall. Phys. Rev. E 79 (2009) 045302.

[23] E.M. Purcell, Life at low Reynolds number. Am. J. Phys. 45 (1977) 3-11.

[24] S.H. Rad and A. Najafi, Hydrodynamic interactions of spherical particles in a fluid confined by a rough no-slip wall. Phys. Rev. E 82 (2010) 036305.

[25] L. Rothschild, Non-random distribution of bull spermatozoa in a drop of sperm suspension. Nature (1963).

[26] D.J. Smith and J.R. Blake, Surface accumulation of spermatozoa: a fluid dynamic phenomenon. Math. Sci. 34 (2009) $74-87$.

[27] D.J. Smith, E.A. Gaffney, J.R. Blake and J.C. Kirkman-Brown, Human sperm accumulation near surfaces: a simulation study. J. Fluid Mech. 621 (2009) 289-320.

[28] G. Taylor, Analysis of the swimming of microscopic organisms. Proc. R. Soc. London A 209 (1951) 447-461.

[29] R. Temam, Navier-Stokes Equations: Theory and Numerical Analysis. AMS, Chelsea (2005).

[30] E.F. Whittlesey, Analytic functions in Banach spaces. Proc. Amer. Math. Soc. 16 (1965) 1077-1083.

[31] H. Winet, G.S. Bernstein and J. Head, Observation on the response of human spermatozoa to gravity, boundaries and fluid shear. Reproduction 70 (1984).

[32] H. Winet, G.S. Bernstein and J. Head, Spermatozoon tendency to accumulate at walls is strongest mechanical response. J. Androl. (1984).

[33] C. Ybert, C. Barentin, C. Cottin-Bizonne, P. Joseph and L. Bocquet, Achieving large slip with superhydrophobic surfaces: scaling laws for generic geometries. Phys. Fluids 19 (2007). 